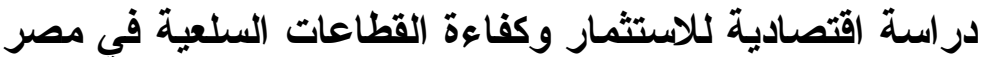

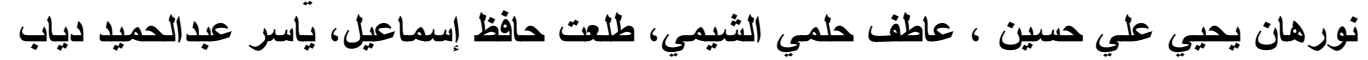

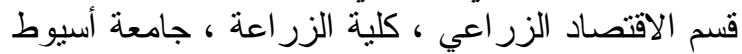

تعتبر الإستثمار ات و القدرة علي توفير ها بالقدر المطلوب في كل القطاعــات الأقتــــادية

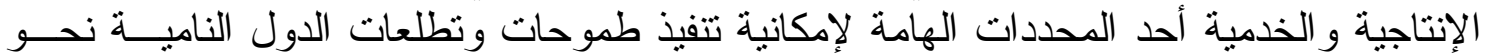

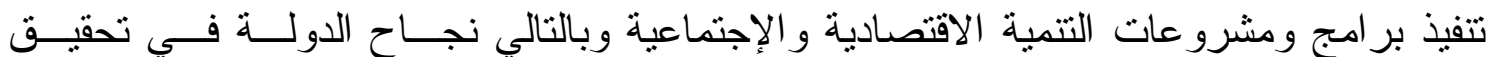

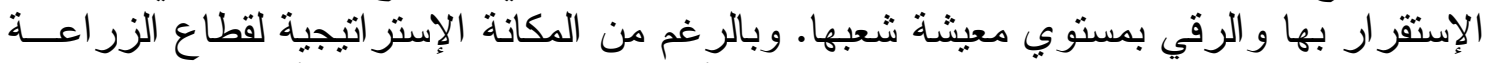

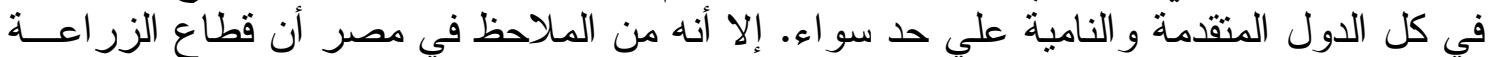
يبدو أنه غير محفز للاستثمار بالمقارنة بباقي القطاعات الاقتصادية كالصناعة و التجارة و التنتييد

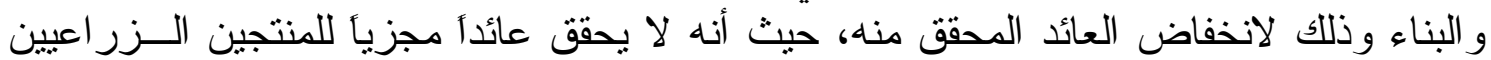

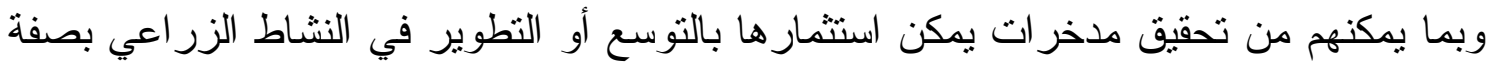

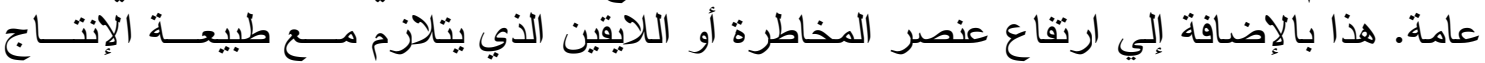

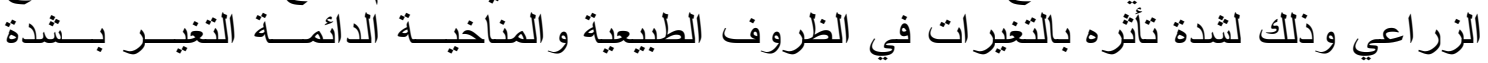

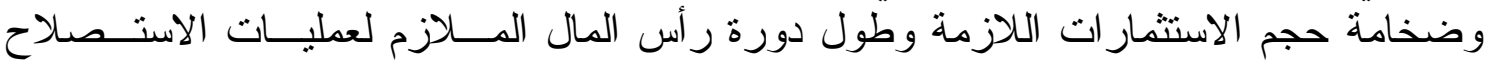

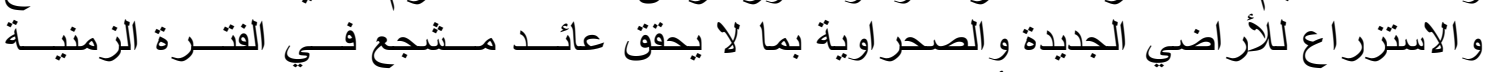

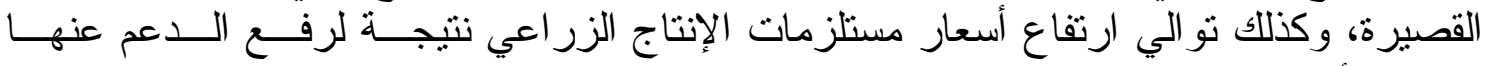

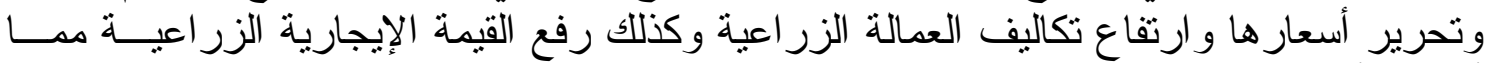

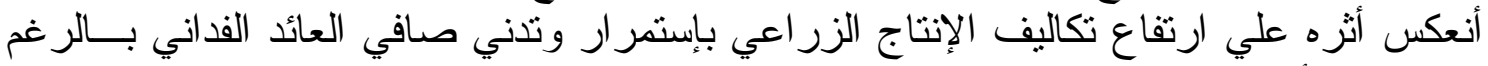

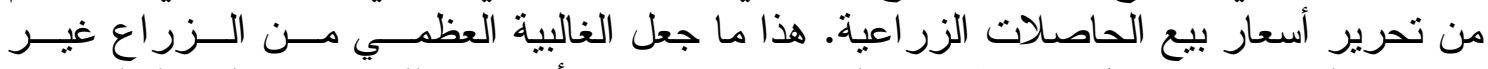

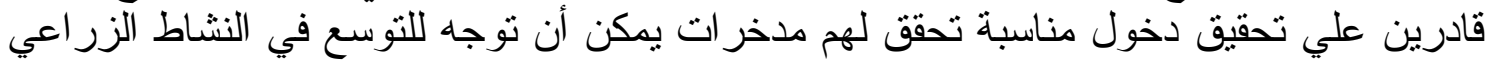
بتوجيه الاستثمار ات له له مشكلة البحث: البه:

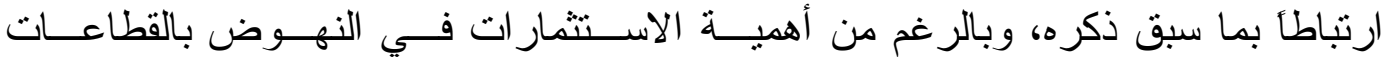

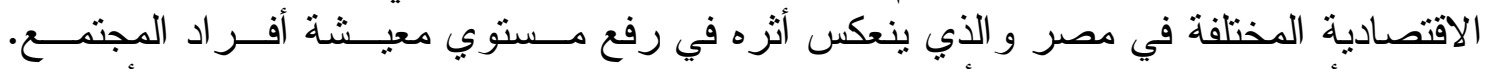

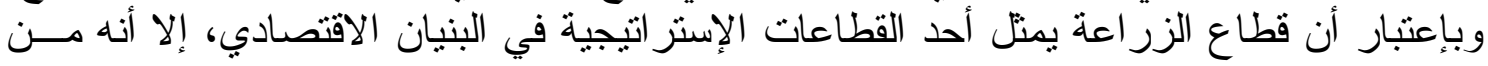

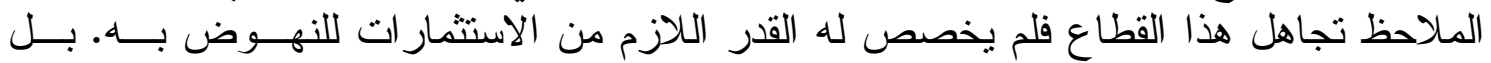

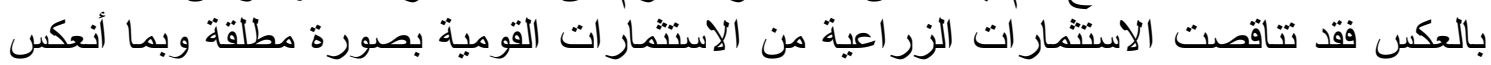

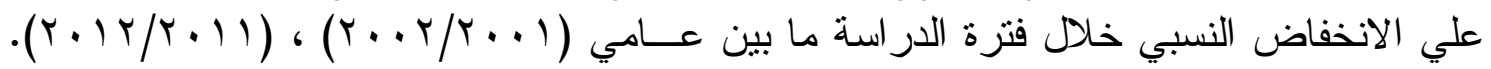

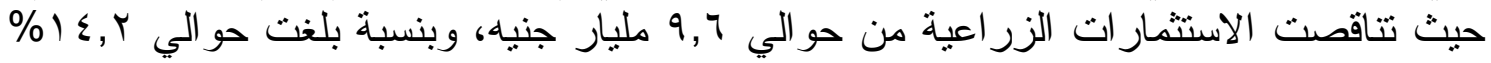

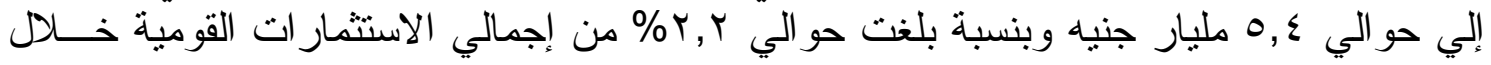

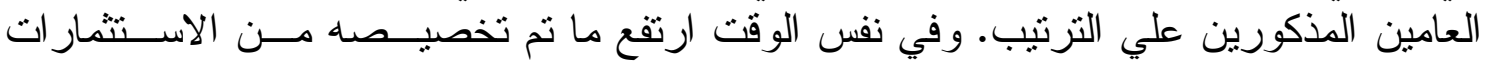

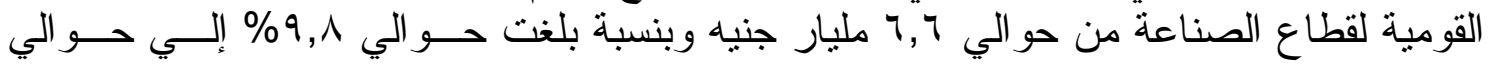

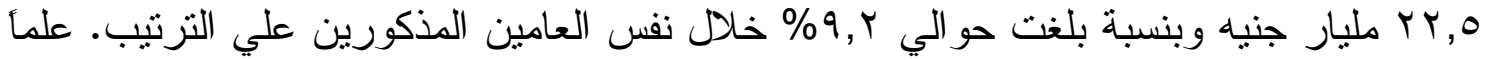

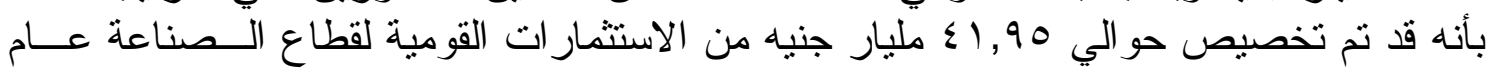

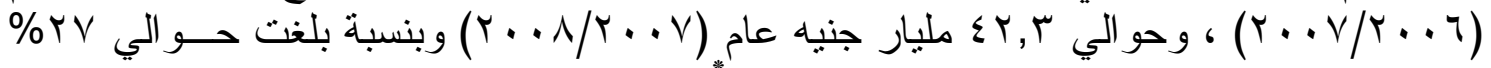

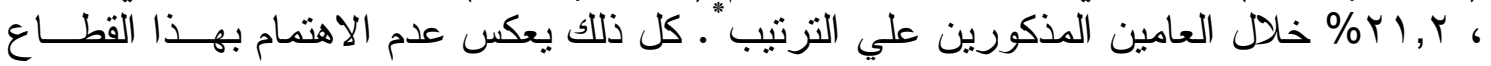
الحيوي.

Received on: 10/4/2014 Accepted for publication on: 15/4/2014

Referees: Prof. Mohamed A. Abonahol Prof. Abdel-wakeel I. Mohamed 
هدف الار استة:

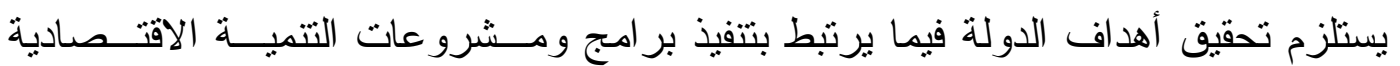

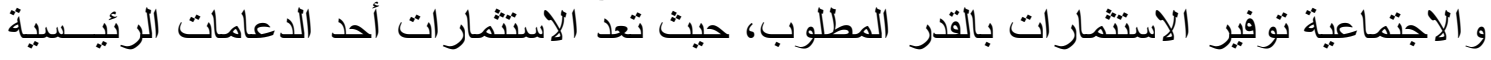

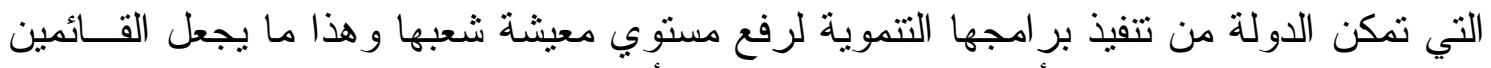

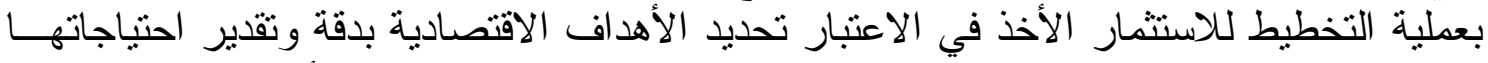

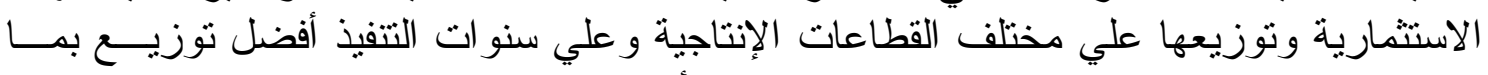

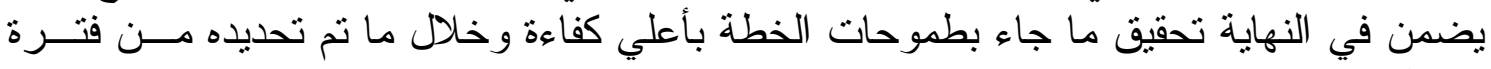
زمنية.

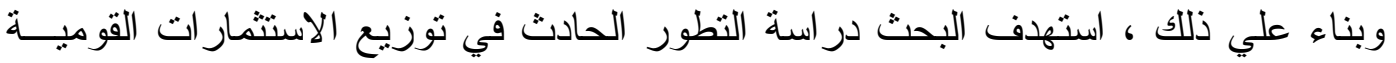

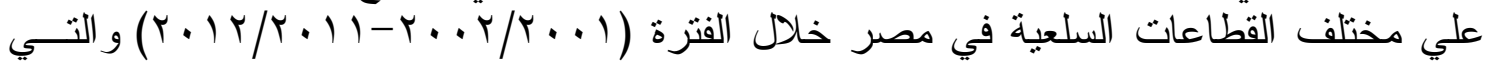

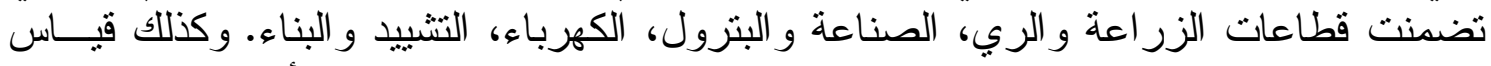

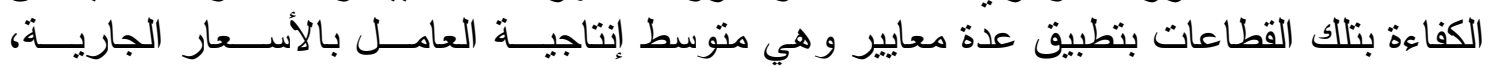

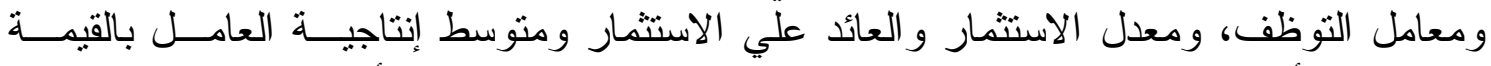

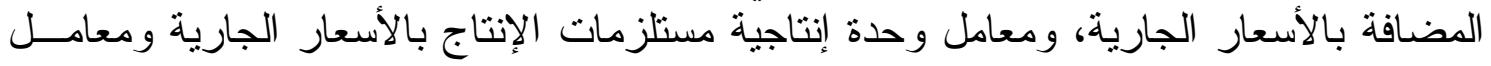

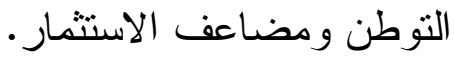
مصادر البيانات:

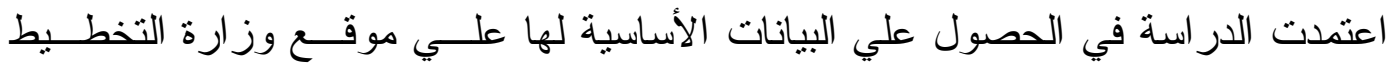

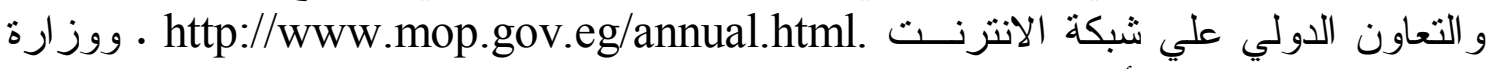

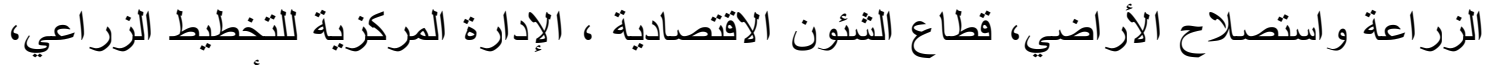

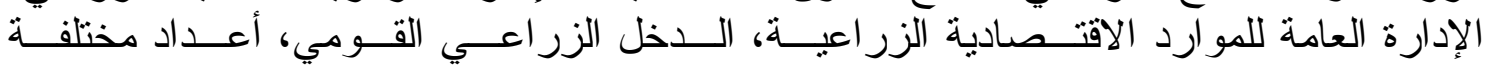

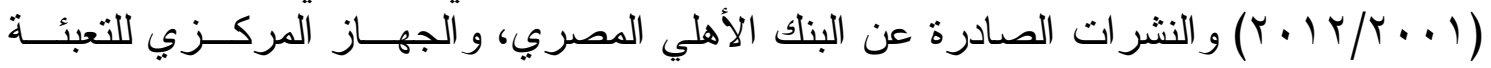

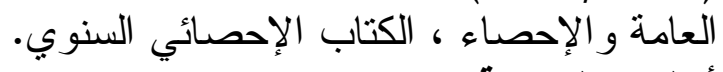

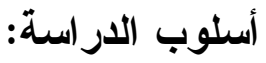

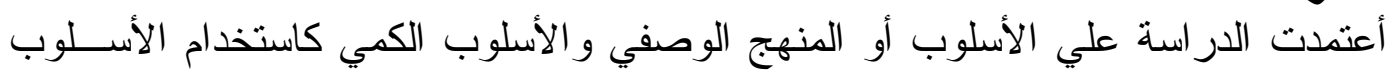

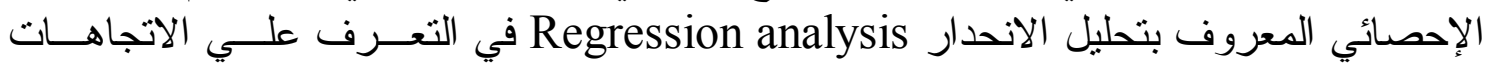

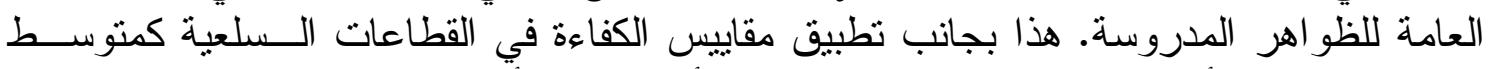

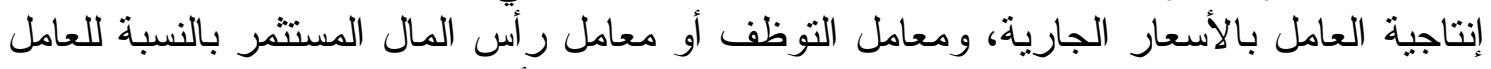

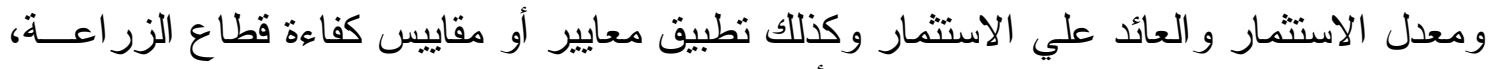

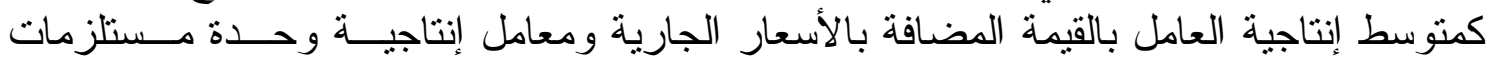

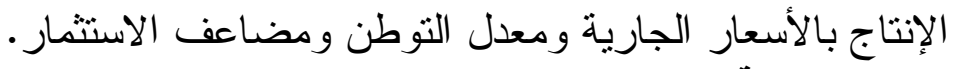
نتائج الار استة: بالأنعار

\section{أولاً: هيكل مكونات الإستثمار ات القومية الإجمالية (العام والخاص) في مصر:}

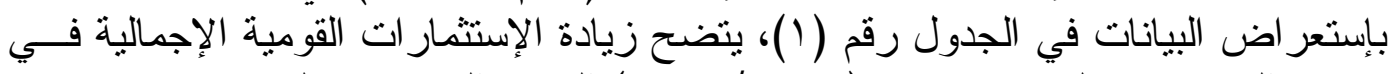

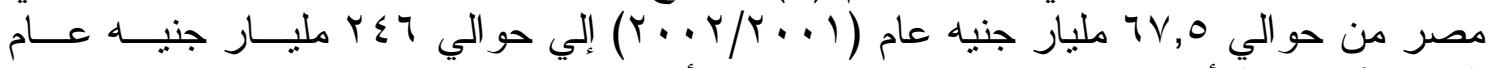

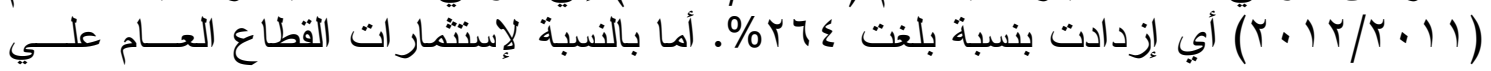

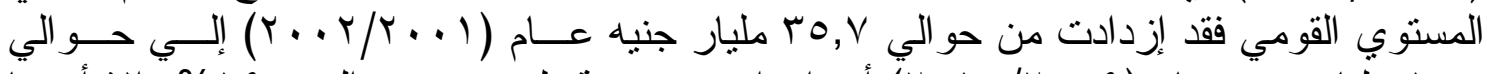

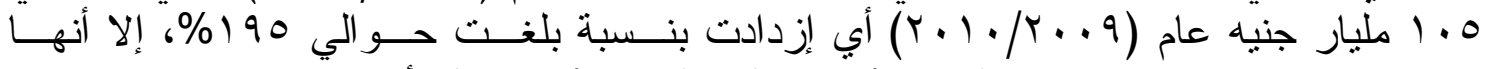

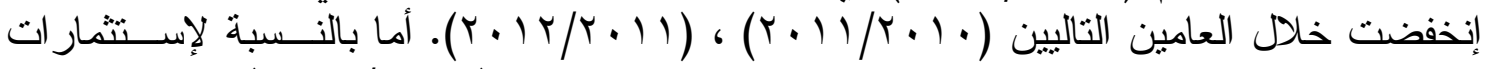

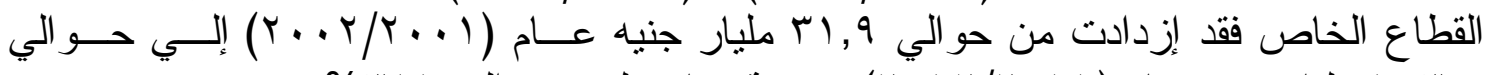

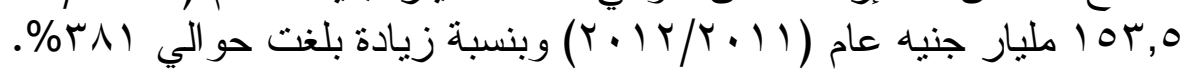

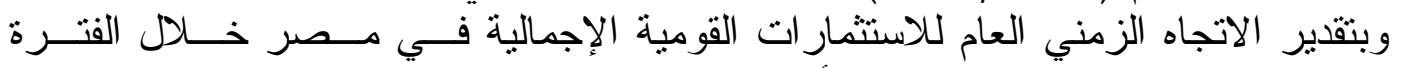

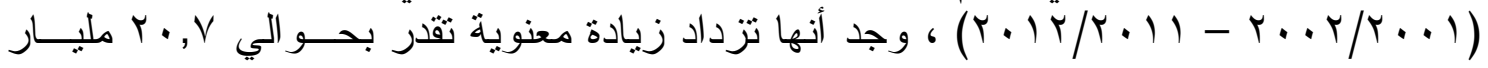

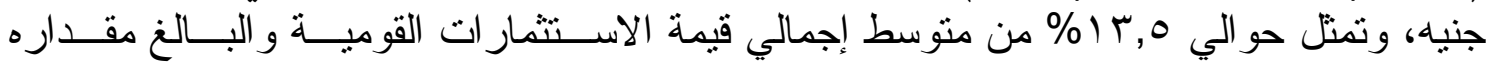




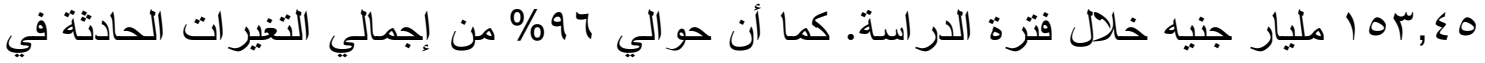

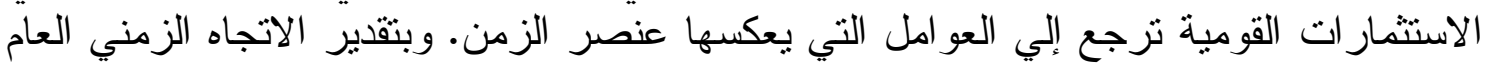

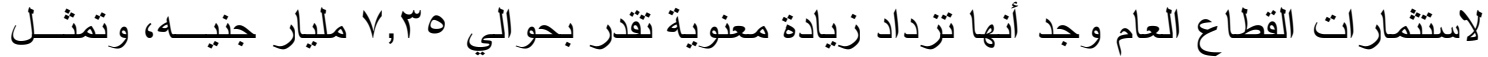

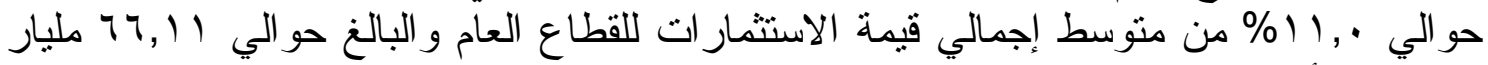

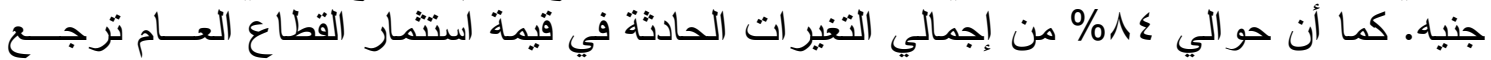

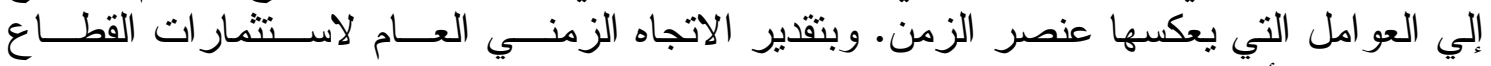

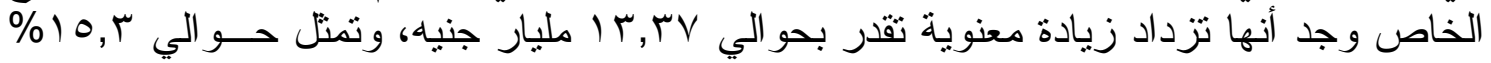

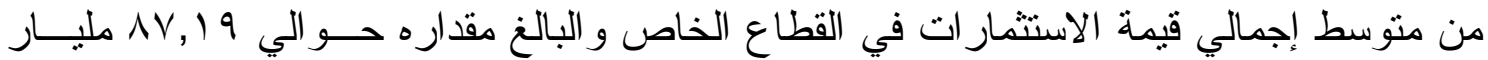

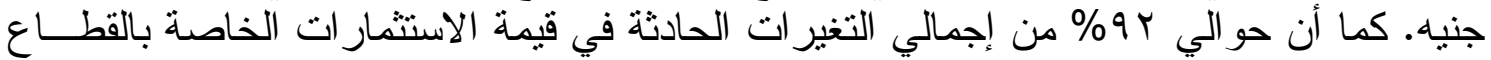

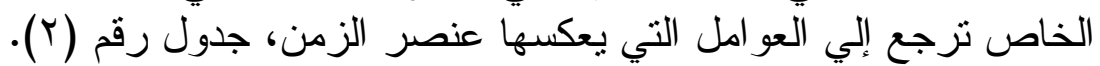

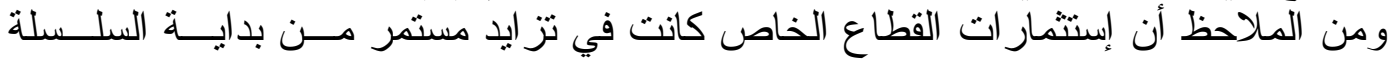

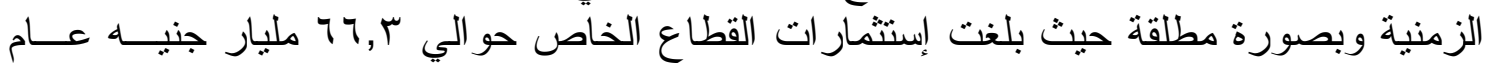

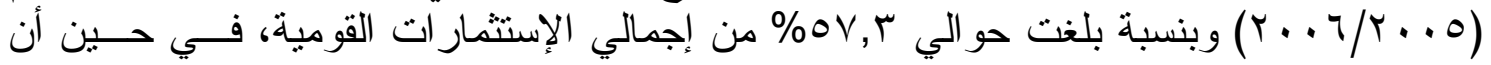

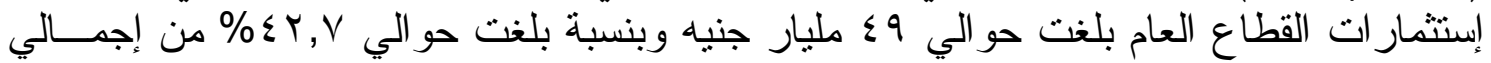

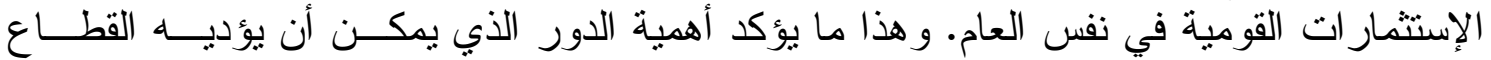

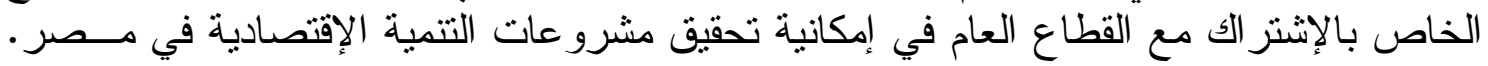

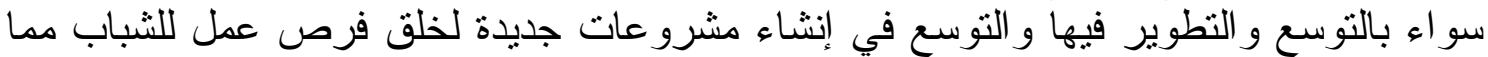

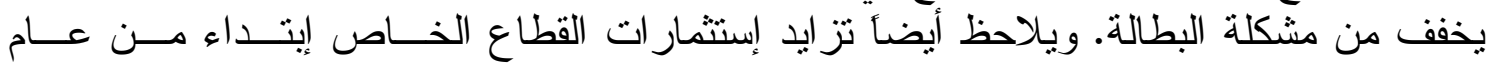

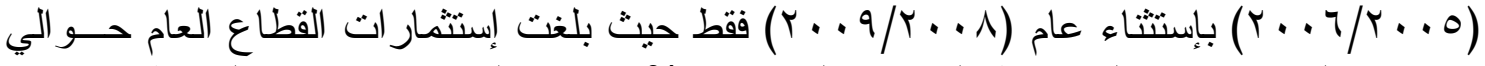

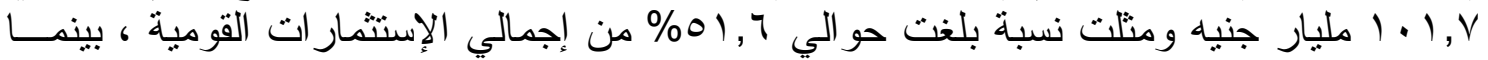

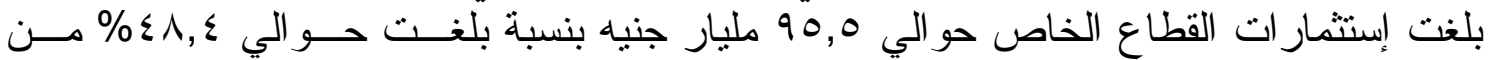
إجمالي الإستثمار ات القومية.

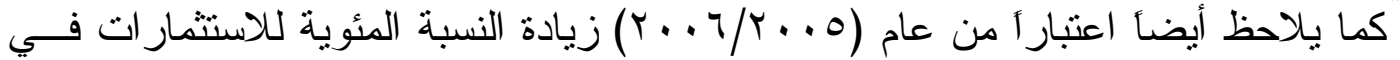

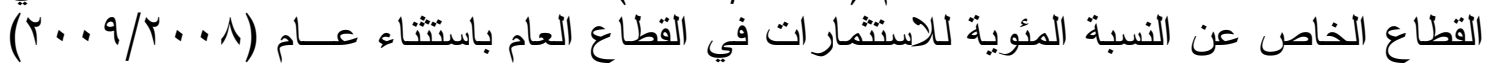

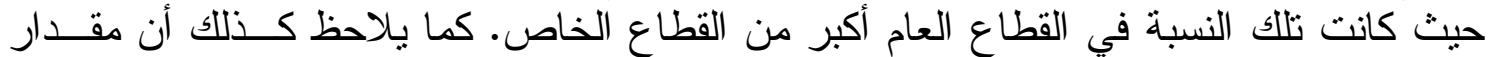

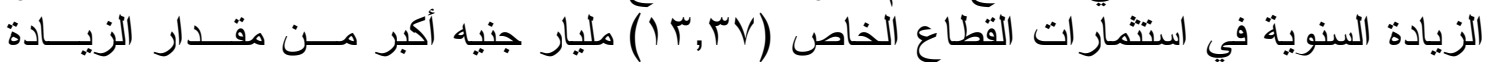

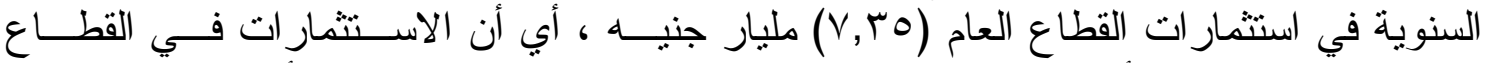

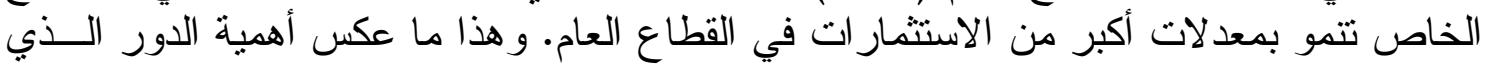
يؤديه القطاع الخاص في الاقتصاد القومي المصري. 


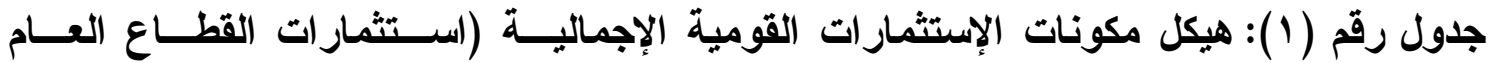

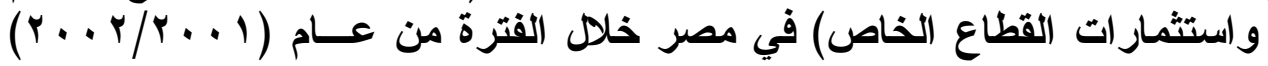

(بالأسعار الجارية بالمليار جنيه)

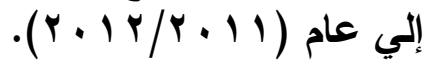

\begin{tabular}{|c|c|c|c|c|c|}
\hline$\%$ & القطاع الخاص اتثمار & $\%$ & القطاع العام & الإستثمارات القومية & السنوات \\
\hline$\varepsilon V, r$ & $\Gamma 1, \wedge \leqslant 7$ & or,,$\Lambda$ & 10,770 & $7 V, 011$ & $r \ldots r / r \ldots l$ \\
\hline$\varepsilon 9, \varepsilon$ & $r r, T \leqslant 7$ & $0 \cdot, 7$ & $\Gamma \varepsilon, \varepsilon O V$ & $7 \Lambda, 1 \cdot r$ & $r \ldots r / r \ldots r$ \\
\hline$\varepsilon 7,7$ & $r v, 1 \ldots$ & Or, ₹ & $\{Y, \leqslant 0\}$ & $V 9,007$ & $r \ldots \varepsilon / r \ldots r$ \\
\hline$\varepsilon \wedge, 1$ & $\sum \neg, \varepsilon \backslash \vee$ & 01,9 & $0 ., \cdot r q$ & $97,\{07$ & $r \ldots o / r \ldots \varepsilon$ \\
\hline$O V, r$ & TY,TYO & $\varepsilon r, V$ & $\{9,\{17$ & $110, v \leqslant 1$ & $r \ldots T / Y \ldots 0$ \\
\hline $7 Y, 7$ & $q \vee, r \ldots$ & $r V, \varepsilon$ & $0 \wedge, \cdot \sum Y$ & $100, r \leqslant Y$ & $r \ldots V / r \ldots r$ \\
\hline $7 \varepsilon, V$ & $1 Y q, \cdot 1$. & $r o, r$ & $V \cdot, \leqslant 00$ & 199,010 & $r \ldots \wedge / r \ldots v$ \\
\hline$\sum \wedge, \varepsilon$ & $90, \leqslant \vee 7$ & 01,7 & $1 \cdot 1,771$ & $19 V, 1 T V$ & $r \ldots q / r \ldots r$ \\
\hline $0 \leqslant, V$ & IY $Y, \vee T \wedge$ & $\varepsilon 0, r$ & $1.0,119$ & YMI,AYV & $r \cdot 1 \cdot / r \ldots q$ \\
\hline 71,9 & $1 \leq 1,7 \vee 7$ & r^, I & $\Lambda \vee, r q$. & YYq,.77 & $r .11 / r \cdot 1$. \\
\hline$\pi r, \varepsilon$ & IOT,OYT & $r V, r$ & $9 Y, 0 \leq 7$ & $r \leq 7, \cdot 7 \wedge$ & $r \cdot 1 r / r \cdot 11$ \\
\hline $0 \leqslant, \wedge 0$ & $\Lambda V, 19$ & $\leqslant 0,10$ & 77,11 & $10 r, \cdot \varepsilon$ & المتوسط \\
\hline
\end{tabular}

المصدر : جمعت وحسبت من:

موقع وز ارة التخطيط و التعاون الدولي علي شبكة الانترنت: http:/www.mop.gov.eg/annual.html

جدول رقم (Y): معادلات الاتجاه الزمني العام لمكونات الاستثمارات القومية الإجمالية بالمليــار

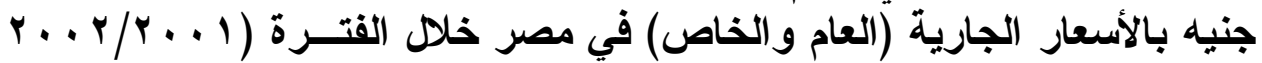

\begin{tabular}{|c|c|c|c|c|c|c|c|}
\hline التغنير & (مقار التغير & 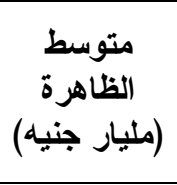 & $\mathbf{F}$ & $\mathbf{R}^{2}$ & $\mathbf{R}$ & معادلة الاتجاه الزمني العام & 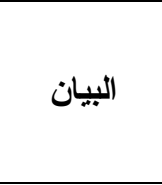 \\
\hline $\mid r, 01$ & $r \cdot, V Y$ & $10 \%, \cdot \varepsilon$ & $* * Y, \leq, \vee q$ & $\cdot, 97$ & $\cdot, 91$ & 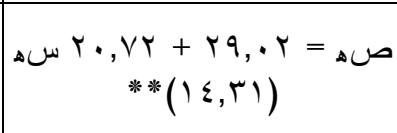 & الإستثمار ات القومية \\
\hline $11,1 r$ & $v, r_{0}$ & 77,11 & $* * \varepsilon \wedge, \varepsilon 1$ & $\cdot, \wedge \varepsilon$ & $\cdot, 94$ & 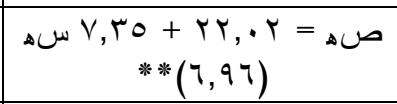 & القطاع العام \\
\hline $10, \pi r$ & IT,rv & $\wedge V, 19$ & $* * 1,1, \leq 9$ & $\cdot, 94$ & $\cdot, 97$ & 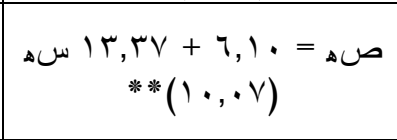 & الختشار ات الخاص ال \\
\hline
\end{tabular}

حيث صهـ تثتير إلي القيمة التقديرية للاستثمار ات القومية الإجمالية واستثمار ات القطاع العام واســتثمار ات القطــاع الخاص بالمليار جنيه.

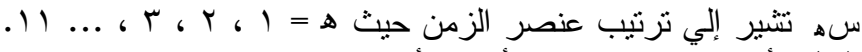

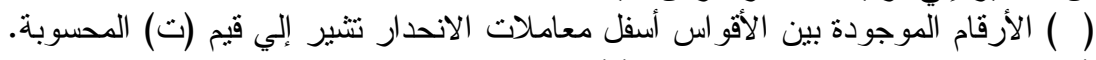

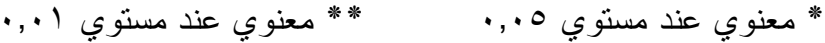
المصدر : جمعت وحسبت من البيانات الو اردة بالجدول رقم (1).

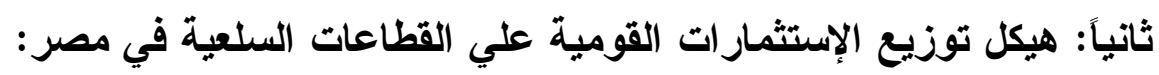

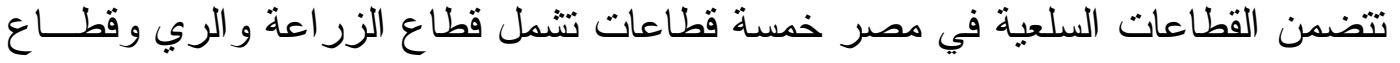

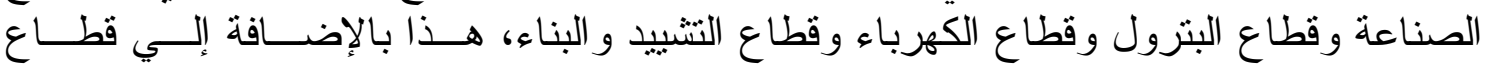

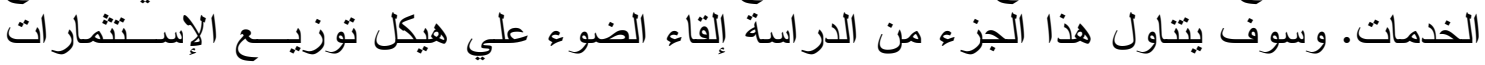

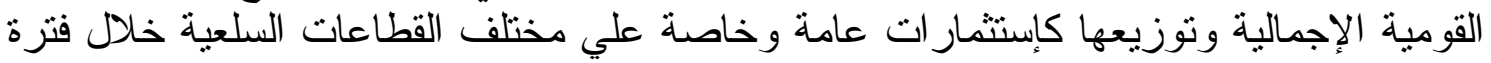

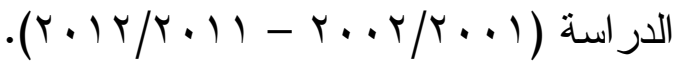


وفي ضوء ما سبق ذكره، توضح البيانات الواردة في الجدول رقـــ (ب) هيكـل توزيــع

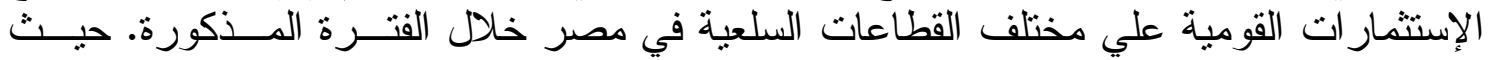

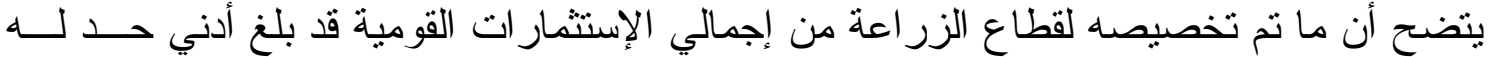

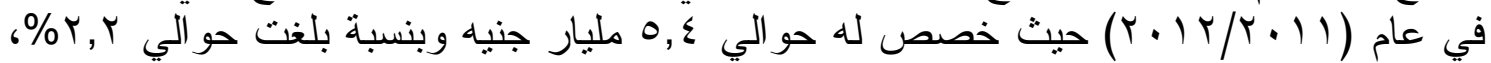

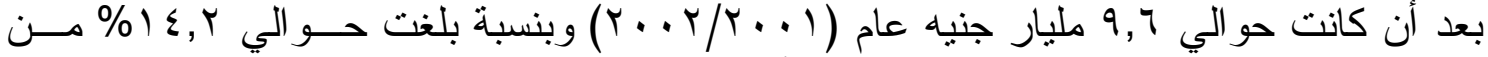

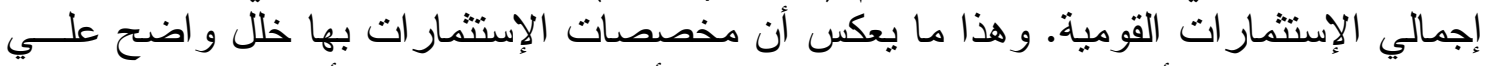

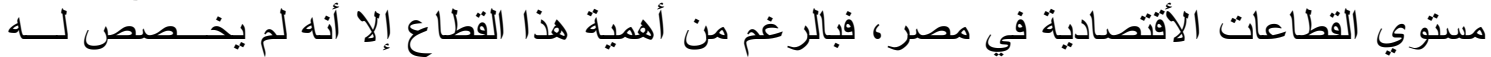

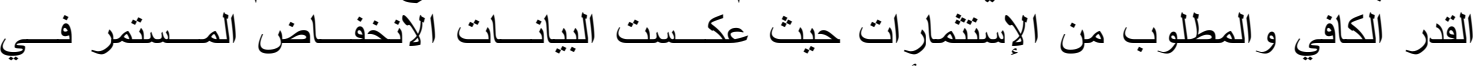
الإستثمار ات بصورة مطلقة وهذا ما أنعكس علي الإنخفاض النسبي المستمر خلال فترة الدر اسة.

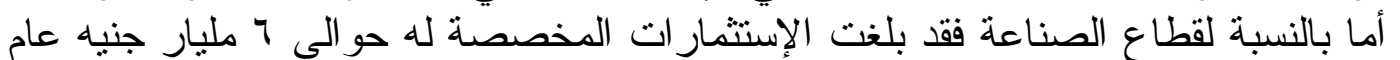

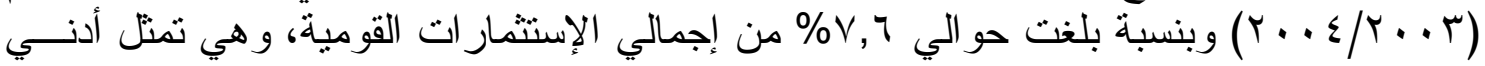

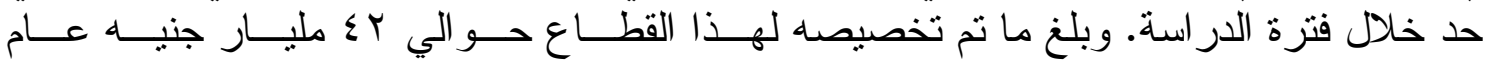

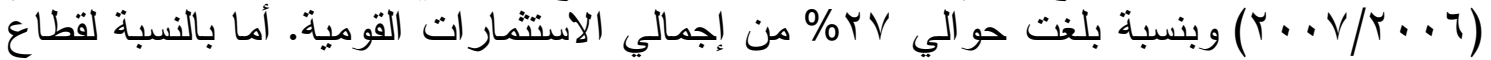

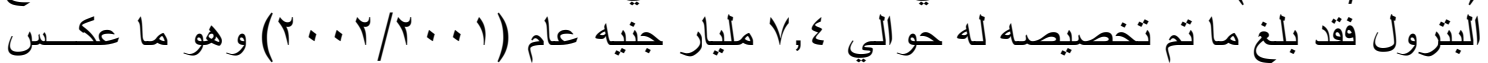

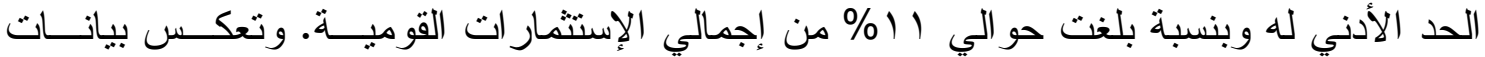

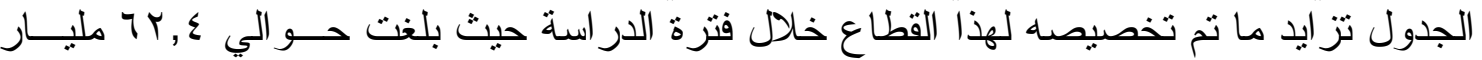

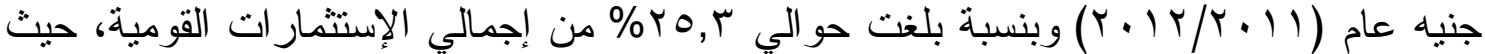

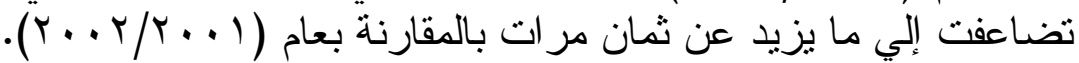

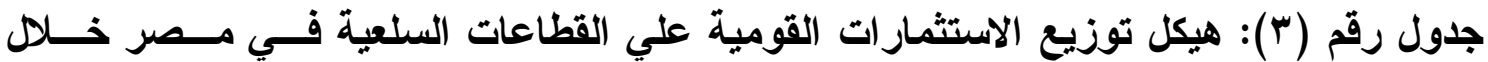

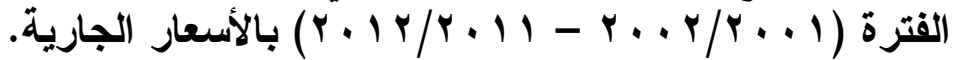

(بالمليار جنيه)

\begin{tabular}{|c|c|c|c|c|c|c|c|c|c|c|c|}
\hline \multicolumn{2}{|c|}{ قالتشييد والبناء } & \multicolumn{2}{|c|}{ قطاع الكهرباء } & \multicolumn{2}{|c|}{ قطاع البترول" } & \multicolumn{2}{|c|}{ قطاع الصناعة } & \multicolumn{2}{|c|}{ 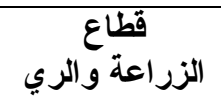 } & \multirow{2}{*}{ الالاستثمارات } & \multirow[t]{2}{*}{ السنوات } \\
\hline$\%$ & الإجمالي & $\%$ & الإجمالي & $\%$ & الإجمالي & $\%$ & الإجمالي & $\%$ & الإجمالي & & \\
\hline$r, \Lambda$ & $1,91$. & $\begin{array}{l}, Y \\
\end{array}$ & $\varepsilon, \wedge \leqslant V$ & $1, \ldots$ & $V, \varepsilon r V$ & $9, \wedge$ & 7,701 & $|\varepsilon, Y|$ & $9,09 \leqslant$ & $7 V, 011$ & $\ldots r / r \ldots l$ \\
\hline$r, \Lambda$ & $1,9 \cdot v$ & $0, V$ & $r, q 1$. & $1 Y, T$ & $\wedge, 0 \wedge 9$ & $1 \cdot, \mathrm{NV}$ & $V, \varepsilon \cdot 1$ & $9, \varepsilon$ & $7, \varepsilon \cdot \varepsilon$ & $T \Lambda, 1 \cdot r$ & $r \ldots r / r \ldots r$ \\
\hline 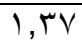 & $1, \cdot 19$ & $9, Y \varepsilon$ & $V, r \leq q$ & 10,91 & $1 Y, V 17$ & $V, 09$ & $7,+\leqslant 0$ & $9,0$. & $V, 009$ & $V 9,007$ & $r \cdots \varepsilon / r_{\cdots}$ \\
\hline Tr & $1,1 \wedge 9$ & $\Lambda, r$ & $v, 901$ & $I Y, V$ & TY,YYq & $T r, I \wedge V$ & $I Y, V Y$. & 8,79 & $V, \Sigma Y$. & $97, \leqslant 07$ & r..o/ $\ldots \ldots$ \\
\hline, $\mathrm{OV}$ & $\varepsilon, 1) \tau$ & $7, \wedge 7$ & $V, q \leqslant 1$ & $Y Y, Y$ & YY,Y०Y & $1 Y, 0 \leqslant$ & $\mid\{, 0 \mid \leqslant$ & 7,90 & $\Lambda, \cdot \leq \varepsilon$ & $110, v \leqslant 1$ & $r \ldots T / r_{\ldots}$ \\
\hline .09 & $Y, \varepsilon V V$ & $\varepsilon, 9$ & $V, T Y M$ & $1 V, r q$ & $T V, \cdot I T$ & $r v, \ldots$ & $\leqslant 1,901$ & $0, \cdot 1$ & $v, \vee q 1$ & $100, r \leqslant r$ & $r \ldots V / r \ldots T$ \\
\hline 1,77 & $r, r / 0$ & $0, r V$ & $1,, V Y O$ & $1 \Lambda, r$ & $r 7,00 Y$ & 41,19 & $\varepsilon r, r \ldots$ & $\varepsilon, \cdot \varepsilon$ & $\Lambda, \cdot V$ & 199,040 & $r \ldots \wedge / r_{\ldots} . V$ \\
\hline 1,90 & $r, \wedge r v$ & $\gamma, 9$ & $10,0 \vee 0$ & $19, \pi v$ & rی, 19\& & $10, .7$ & 49,791 & $r, \varepsilon \wedge$ & T,Aтr & $19 V, 1 T V$ & $r \ldots q / r \ldots 1$ \\
\hline 1,19 & $\varepsilon, 17 \pi$ & 7,1 & $10, \Lambda T M$ & YY, $\{\varepsilon$ & $71, Y 9 V$ & $11, .11$ & $Y 0,0 \leqslant 0$ & $r, q 1$ & $7, V \leqslant r$ & TrI,ATV & $r \cdot 1 \cdot / r \ldots q$ \\
\hline$r, 0$ & $0, V \backslash Y$ & $V, r 4$ & $17, \wedge \Lambda$. & 19,0 & $\varepsilon \varepsilon, V Y\}$ & $1 \cdot, r V$ & YT,VTV & $r, 91$ & $\neg, \wedge \Gamma \leq$ & YYQ,.TT & $r \cdot 11 / r \cdot 1$. \\
\hline$\cdot, 79$ & 1,799 & $V, \varepsilon \wedge$ & $|\wedge, \varepsilon \cdot|$ & TO, TE & TY,Yרד & 9,10 & YY,OYq & Y,11 & $0, r \vee v 1$ & $T \leqslant \tau, \cdot T \Lambda$ & $T \cdot \mid r / Y \cdot 11$ \\
\hline$Y, \cdots$ & Y,AT & $v, \ldots$ & $1 \cdot, 7 \varepsilon$ & $1 \wedge, r$ & $r \cdot, T V$ & $1 \Gamma, \varepsilon \Gamma$ & 41,19 & $7, Y 1$ & $V, r \varepsilon$ & $10 \%, \cdot 5$ & المتوسط \\
\hline
\end{tabular}

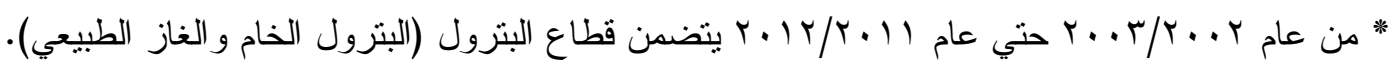

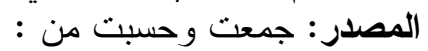

http://www.mop.gov.eg/annual.html موقع وزارة التخطيط و التعاون الدولي علي شبكة الإنترنت

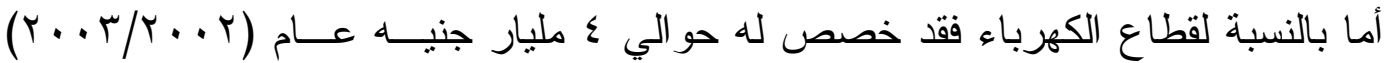

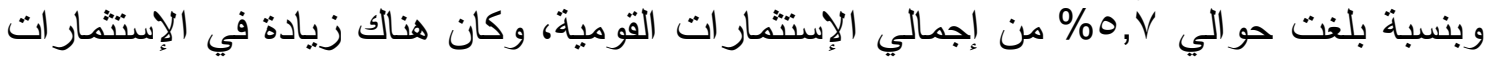

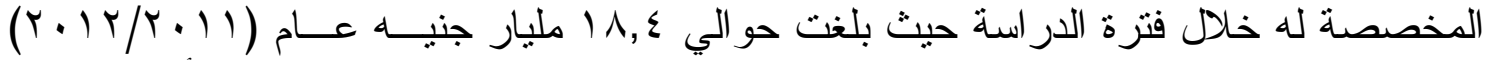

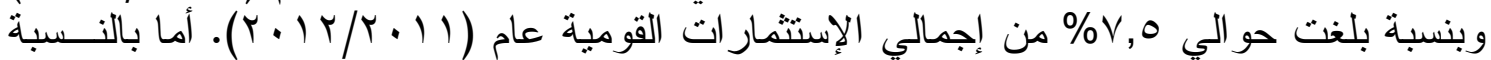

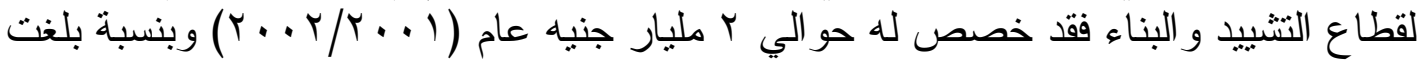




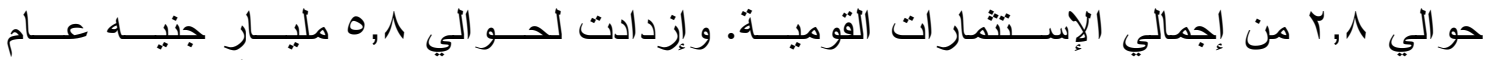

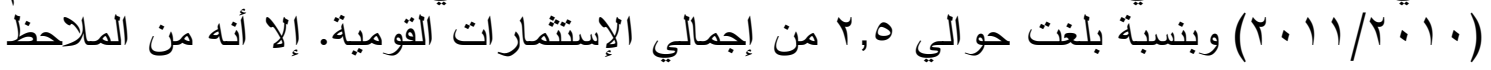

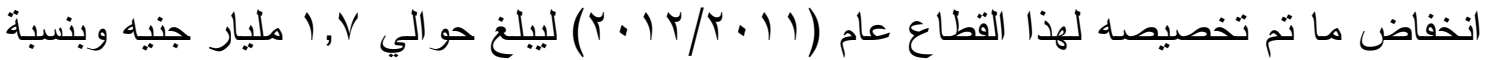

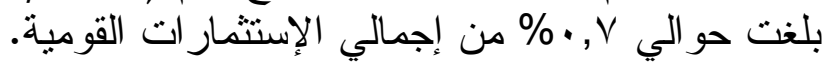

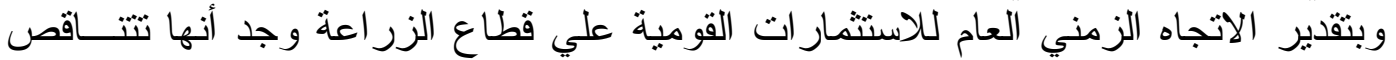

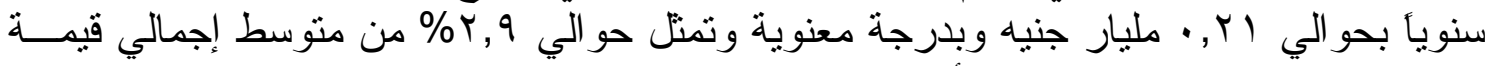

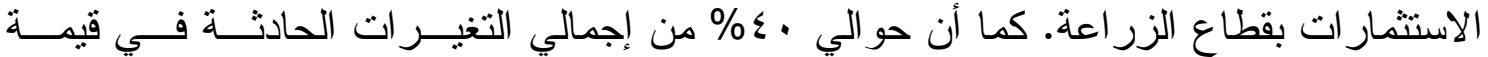

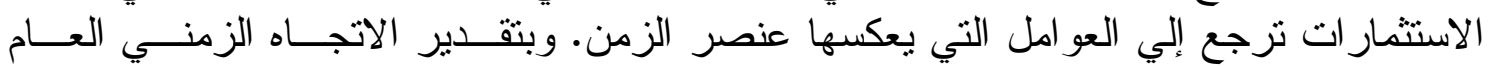

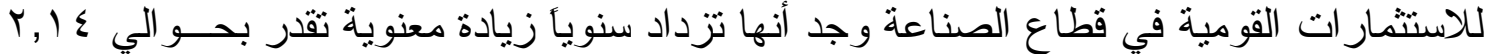

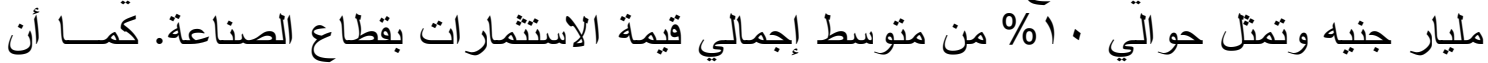

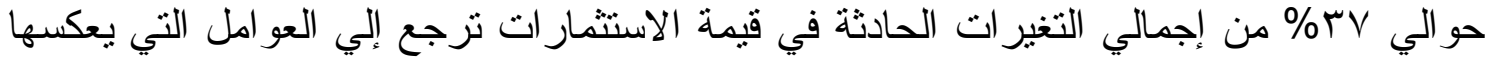

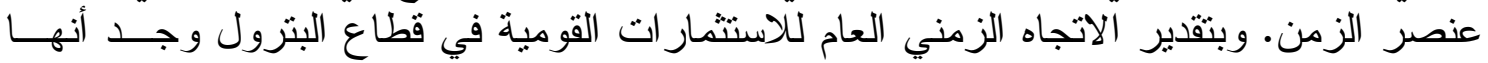

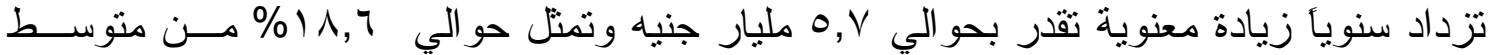

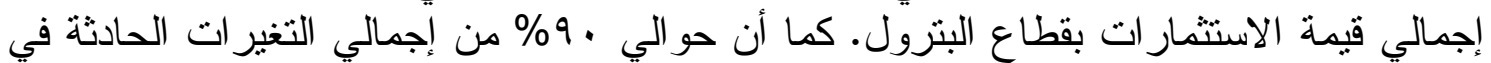

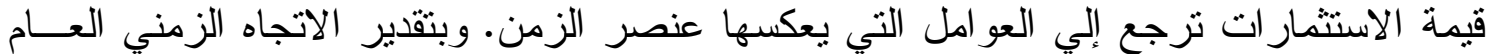

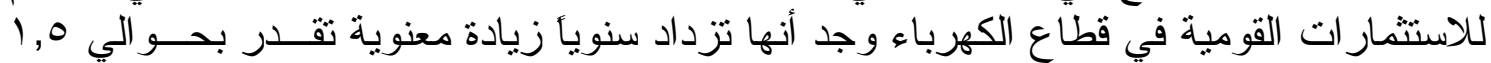

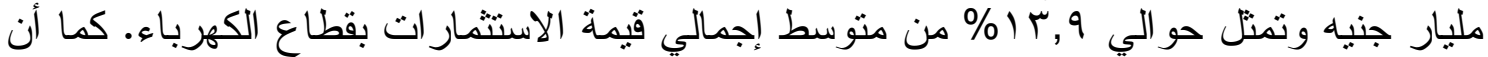

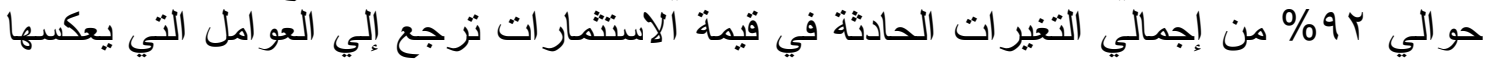

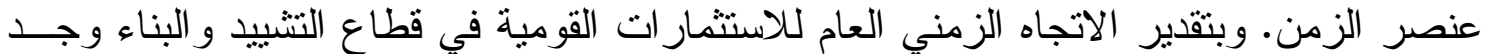

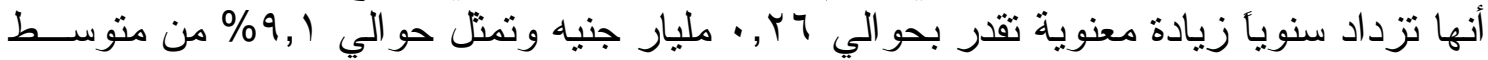

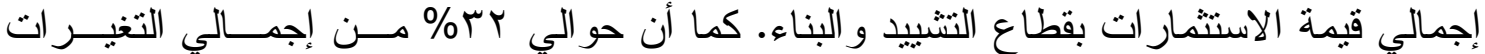

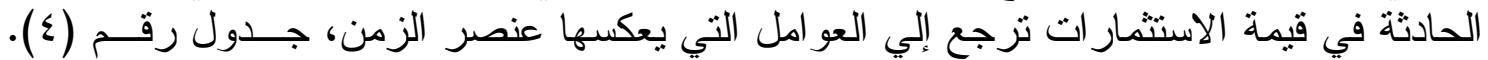

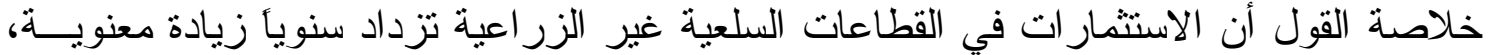

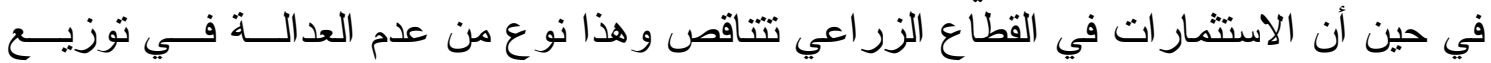

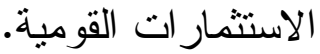

ثالثاً : هيكل توزيع استثمار ات القطاع العام علي القطاعات السلعية في مصر:

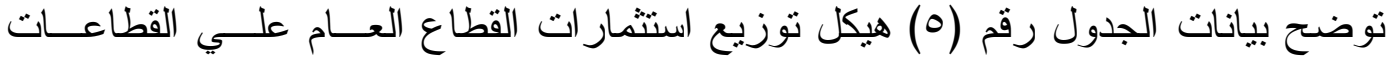

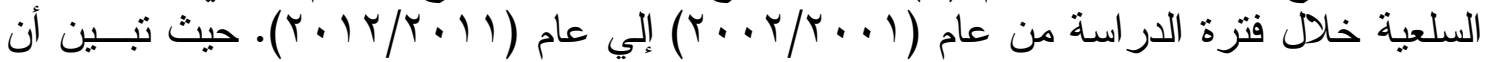

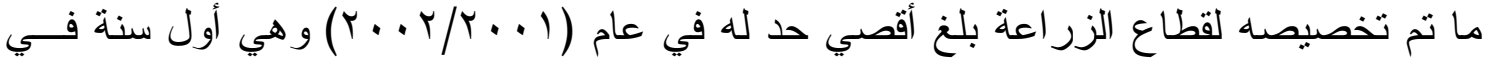

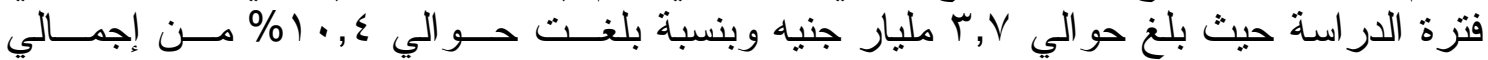
استثمار ات القطاع العام وظلت الإستثمار ات في تتاقص مستمر لتبلغ حو الي Y,V مليار جنيه عام

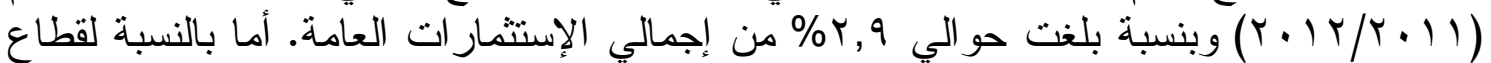

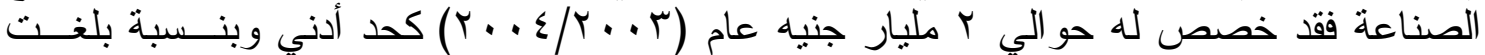

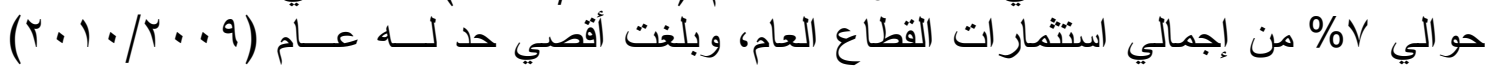

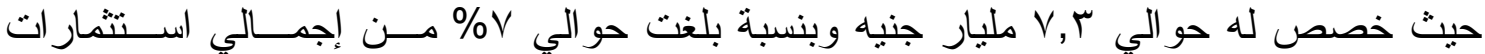

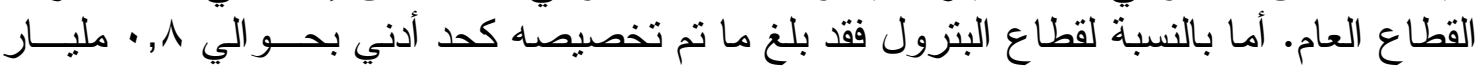

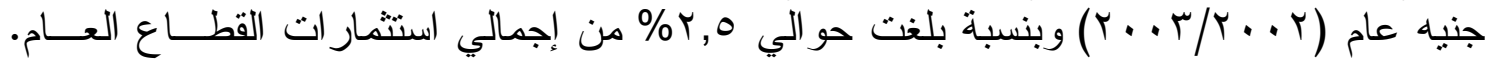

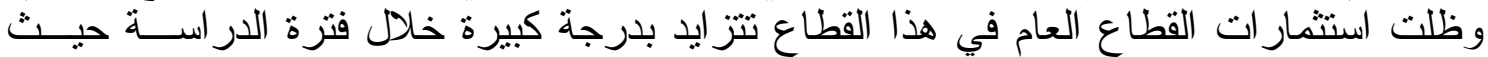

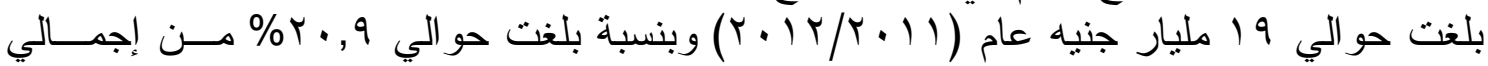
استثمار ات القطاع العام. 


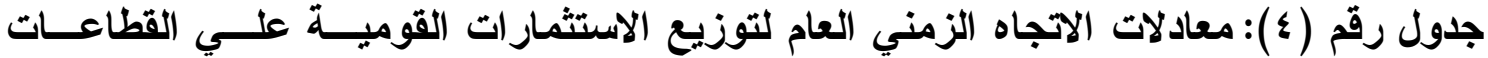

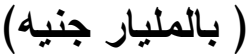

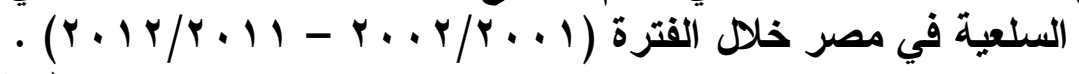

\begin{tabular}{|c|c|c|c|c|c|c|c|}
\hline $\begin{array}{c}\text { التغنير } \\
\text { السنوي } \\
\text { (\%) }\end{array}$ & مقدار التغير & (مليار جنيطه) & $\mathbf{F}$ & $\mathbf{R}^{2}$ & $\mathbf{R}$ & معادلة الاتجاه الزمني العام & البيان \\
\hline$\frac{1}{r, \wedge\urcorner-}$ & $\cdot, Y Y-$ & $V, r \varepsilon$ & $* 0,99$ & $\cdot, \varepsilon \cdot$ & $\cdot, 7 \pi$ & 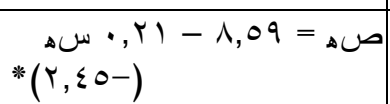 & قطاع الزر اعة \\
\hline $1 \cdot, 1$. & $Y, 1 \varepsilon$ & $r 1,19$ & $* 0, r$ & $\cdot, r V$ & $\cdot, 71$ & $\begin{array}{l}\text { صuه } \\
*(Y, r)\end{array}$ & قطاع الصناعة \\
\hline $\mid \Lambda, \Delta \Lambda$ & $0, v$ & $T \cdot, T V$ & $* * \wedge 0,11$ & $\cdot, 9$. & $\cdot, 90$ & $\begin{array}{l}\text { هu } 0, V \cdot+r, 0 \leqslant-=\Delta \sim ص \\
* *(9, r r)\end{array}$ & قطاع البترول \\
\hline$|r, 9|$ & $1, \varepsilon \wedge$ & $1 \cdot, 7 \varepsilon$ & $* * 99, \varepsilon r$ & $\cdot, 94$ & $\cdot, 97$ & 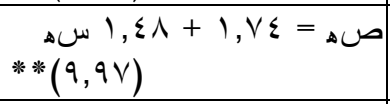 & قطاع الكهرباء \\
\hline $9, .9$ & •, Yד & Y,AT & $* \xi, r$ & $\cdot, r Y$ & $\cdot, \mathrm{OV}$ & 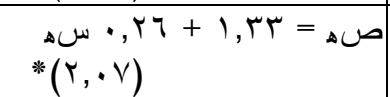 & قطاع التشييد \\
\hline
\end{tabular}

حيث صه تشير إلي القيمة التقديرية للاستثمار ات بالمليار جنيه بمختلف القطاعات الاقتصادية في مصر ، القراء

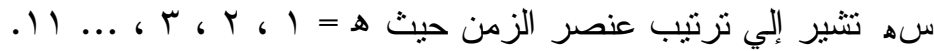

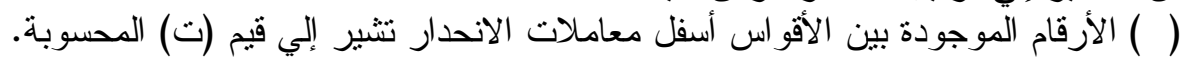

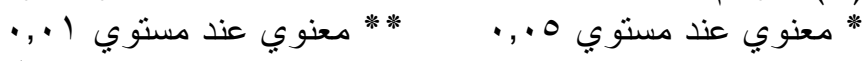

المصدر: جمعت وحسبت من البيانات الو أردة بالجدول رئ رقم (r).

وبالنسبة لقطاع الكهرباء فقد بلغ أدني حد لما خصص من استثمار ات القطاع العام في عام

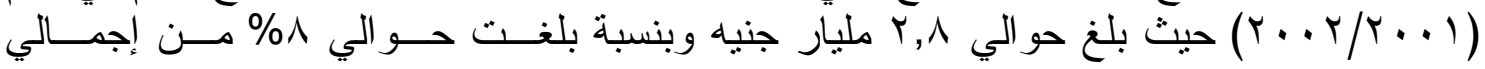

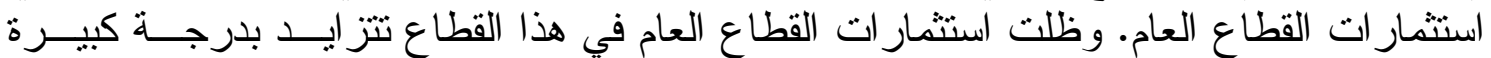

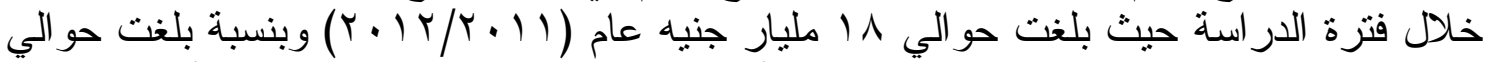

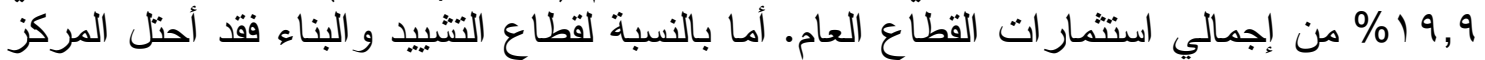

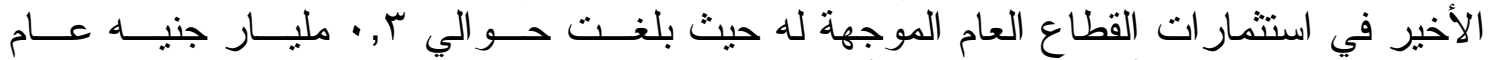

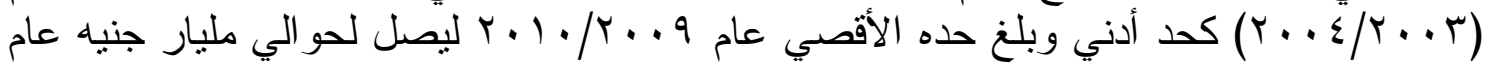

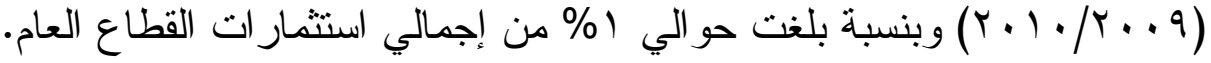

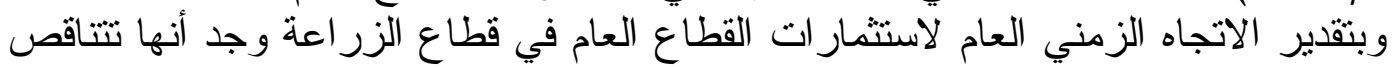

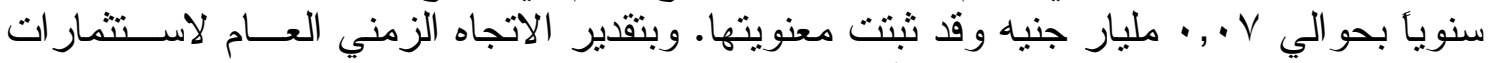

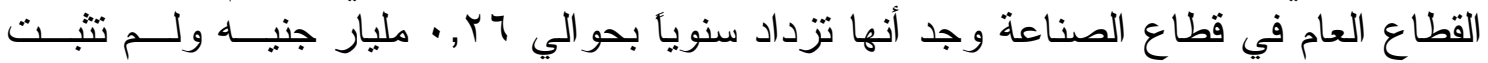
معنويتها.

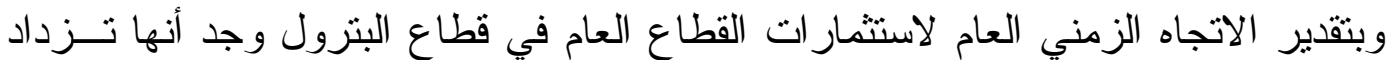

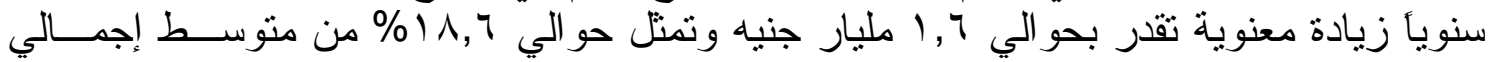

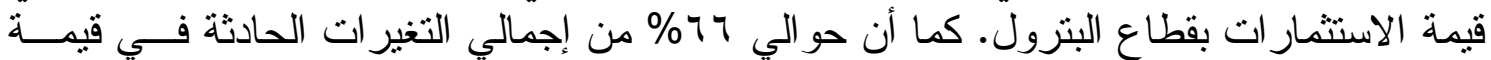

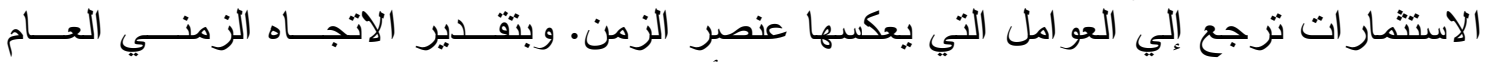

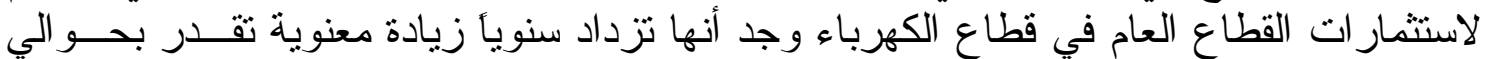

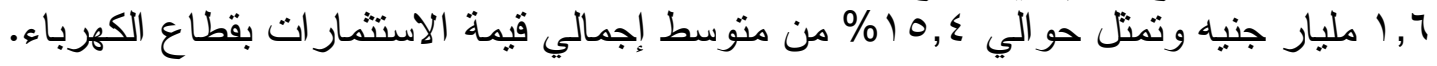




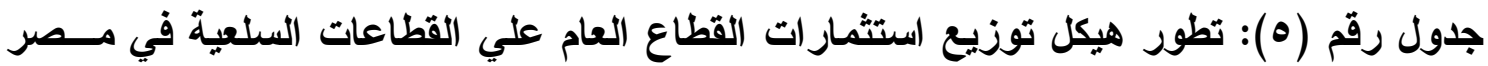

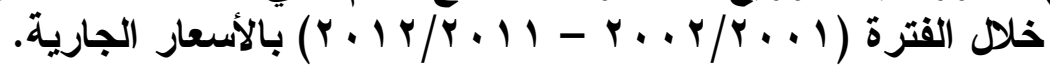

(بالمليار جنيه)

\begin{tabular}{|c|c|c|c|c|c|c|c|c|c|c|c|}
\hline \multicolumn{2}{|c|}{ قطاع التشيياء } & \multicolumn{2}{|c|}{ قطاع الكهرباء } & \multicolumn{2}{|c|}{ قطاع البترول } & \multicolumn{2}{|c|}{ قطاع الصناعة } & \multicolumn{2}{|c|}{ قطاع الزراعة } & \multirow{2}{*}{ القطتثـار ات العام } & \multirow[t]{2}{*}{ السنوات } \\
\hline$\%$ & الإجمالي & $\%$ & الإجمالي & $\%$ & الإجمالي & $\%$ & الإجمالي & $\%$ & الإجمالي & & \\
\hline $1, \cdot$ & $\cdot$, rVo & $\vee, 9 \vee$ & $r, \wedge \leq 0$ & $11, r V$ & $\varepsilon, \cdot r$. & $9, v$ & $r,\{7)$ & דוr, & $r, 790$ & ro, & $r \ldots r / r \ldots l$ \\
\hline $1, \varepsilon$ & $\cdot, \varepsilon \wedge \vee$ & $9, \Sigma) \vee$ & $r, r \leq 0$ & $Y, \leqslant V$ & $\cdot, \wedge 0 \leqslant$ & V,rT & r,OYT & $q, r$ & T,YY. & $r \varepsilon, \varepsilon \circ V$ & $r \cdots r / r \cdots r$ \\
\hline$\cdot, 7$ & $\cdot, Y \wedge 9$ & $17, \wedge r$ & $v, 1 \leq 9$ & r, & $1, \leqslant \leqslant \wedge$ & $v, \cdot 9$ & $r, \cdot 1 r$ & $\wedge, r \wedge$ & $r, 009$ & $\varepsilon r, \varepsilon$ & $r \ldots \varepsilon / r \ldots r$ \\
\hline$\cdot, V$ & $\cdot, r \circ \leq$ & $10, \wedge \wedge 9$ & $v, 901$ & $1 \cdot, r$. & 0,100 & $9, \cdot$ & $\varepsilon, 01 Y$ & $\tau, \Gamma \varepsilon$ & $r, I V$. & $0 .$, & $r \ldots o / r \ldots \varepsilon$ \\
\hline$\cdot, \wedge$ & $\cdot, \varepsilon r \mid$ & $17, .79$ & $v, q \leq 1$ & 17,95 & $\Lambda, r 4)$ & $1 \cdot, 11$ & $0, \cdot r \varepsilon$ & $0, \sum \wedge$ & $r, r)$. & $\leqslant 9, \varepsilon$ & $r \ldots r / r \ldots o$ \\
\hline$\cdot, \wedge$ & $\cdot, \Sigma \vee \wedge$ & Ir, & $V, T Y I$ & $\mid \wedge, r \wedge$ & $1,-$ & $9, v$ & 0,707 & $\varepsilon, 19$ & $r, \varepsilon r \varepsilon$ & os & $r \ldots v / r \ldots r$ \\
\hline$\cdot, 9$ & $\cdot$, & $10, r$ & 1. & 1 & $\Lambda, r \circ r$ & $\Lambda, \Gamma \varepsilon$ & $0, \wedge \vee V$ & $\varepsilon, 1$ & $r, \wedge \leqslant q$ & , & $r \cdots \wedge / r \ldots v$ \\
\hline$\cdot, 9$ & $\cdot, 9$ & $r$ & vo & 9 & $\varepsilon, 7 \wedge \uparrow$ & $7, \wedge$ & 7,947 & $r, 7 q$ & $r, v \leqslant r$ & 1 & $r \ldots q / r \ldots 1$ \\
\hline $1, \cdot$ & $1, \cdot 7 r$ & $10, .9 \leq$ & 10,人тr & $19, V v$ & $r \cdot, 9 r_{4}$ & 7,90 & $V, r \cdot o$ & $r, v r$ & $r, \wedge \vee \wedge$ & $1 \cdot 0, \cdot 19$ & $r \cdot 1 \cdot / r \cdot . q$ \\
\hline 1,1 & . & 19,410 & $17, \wedge \Lambda$. & $1 r, v \cdot \lambda$ & $11,91$. & 0,7 & $\varepsilon, \wedge \wedge \vee$ & $r, v$ & $r, r \vee T$ & $\wedge \vee, r q$. & $r \cdot 11 / r \cdot 1$. \\
\hline$\cdot, 9$ & $\cdot, \wedge) \cdot$ & $19, \wedge 1$ & $\mid \wedge, \varepsilon \cdot 1$ & $r \cdot, \wedge V$ & $19, \pi 17$ & $r, r q$ & $r, .0 r$ & $r, \wedge q$ & $r, T \vee T$ & $9 r, 0 \leq 7$ & $r \cdot 1 r / r+11$ \\
\hline •, 94 & • & $1 \varepsilon, \wedge r$ & $1 \cdot, \Gamma \wedge$ & $1 \%, \cdot \varepsilon$ & $\wedge, \vee$ & $V, T r$ & $\varepsilon, 77$ & $0, \Sigma V$ & $r, \cdot r$ & 77,11 & المتوسط \\
\hline
\end{tabular}

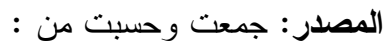
http:/www.mop.gov.eg/annual.html موقع وزارة التخطيط و التعاون الدولي علي شبكة الإنترنت -

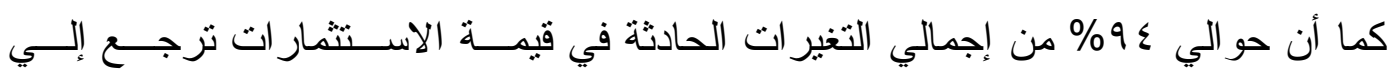

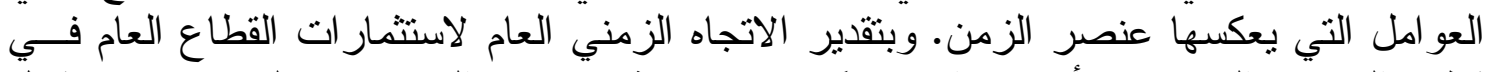

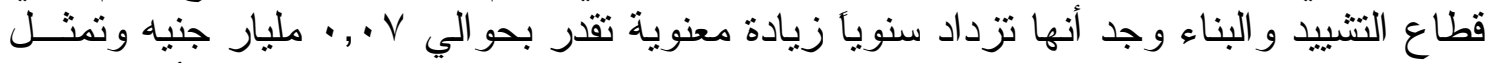

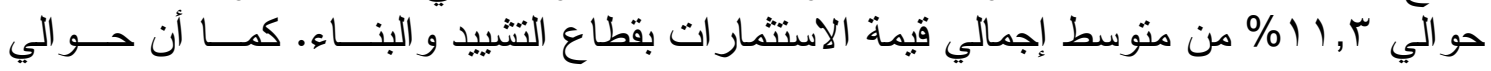

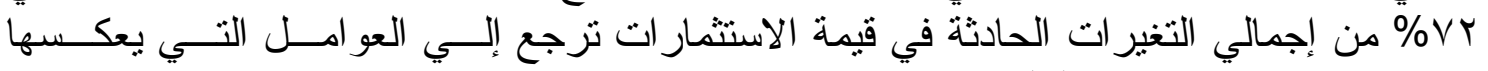

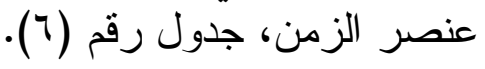
جدول رقم (†): معادلات الاتجاه الزمني العام لاستثمار ات القطاع العام العام علي القطاعات السلعية

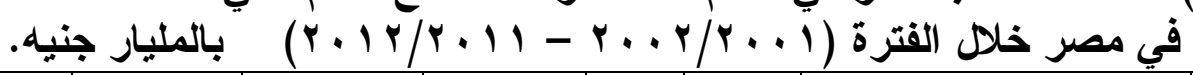

\begin{tabular}{|c|c|c|c|c|c|c|c|}
\hline السنوي (\%) & مقدار التغير (مليار جنيه) & متوسط الظاهرة & $\mathbf{F}$ & $\mathbf{R}^{2}$ & $\mathbf{R}$ & معادلة الاتجاه الزمني العام & البيان \\
\hline Y,YY- & $\cdot, \cdot V-$ & $r, \cdot r$ & $* \varepsilon, 70$ & $\cdot, \Gamma \varepsilon$ & $\cdot, 01$ & 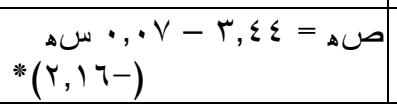 & قطاع الزر اعة \\
\hline 0,01 & $\cdot, Y 7$ & $\varepsilon, 77$ & $r, \cdot 1$ & $\cdot, Y_{O}$ & $\cdot, 0$ & 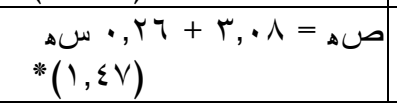 & قطاع الصناعة \\
\hline $1 \Lambda, T Y$ & 1,T & $\Lambda, \nu$ & $* * \mid V, r$ & • & $\cdot, \wedge)$ & 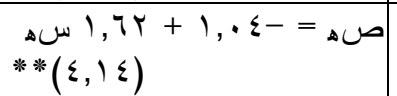 & قطاع البترول \\
\hline $\mid 0, \S 1$ & 1,7 & $1 \cdot, \mathrm{rA}$ & **) $\varepsilon \cdot, \Gamma \uparrow$ & $\cdot, 9 \leqslant$ & $\cdot, 9 V$ & 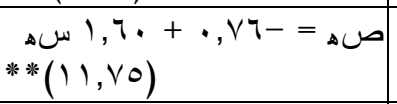 & قطاع الكهرباء \\
\hline 11,19 & $\cdot, \cdot V$ & זד, & $* * r r, \cdot \Lambda$ & $\cdot, V Y$ & $\cdot, \wedge 0$ & 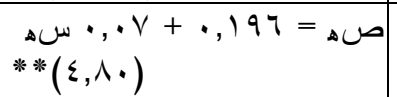 & قطاع التشييد \\
\hline
\end{tabular}

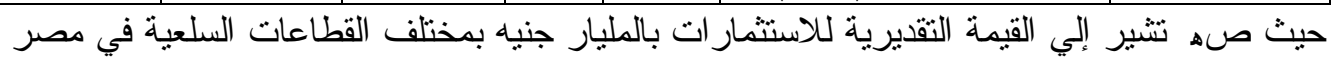

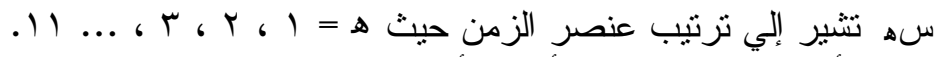

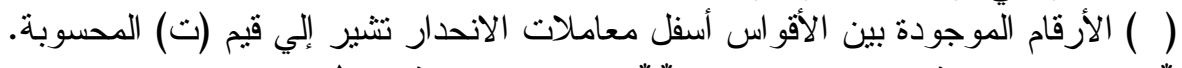

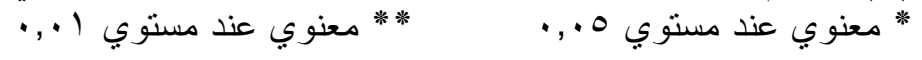


المصدر : جمعت وحسبت من البيانات الو اردة بالجدول رقم (0).

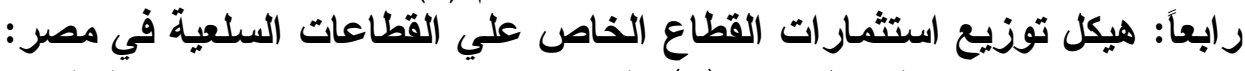

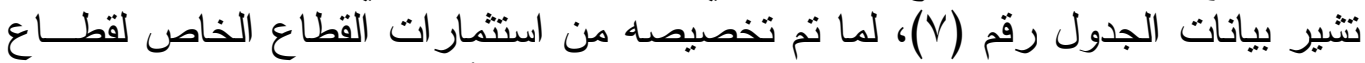

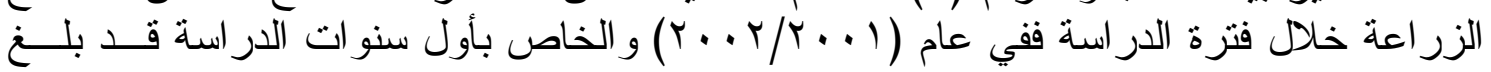

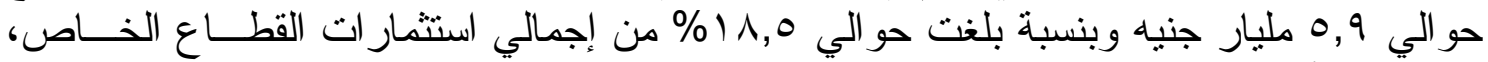

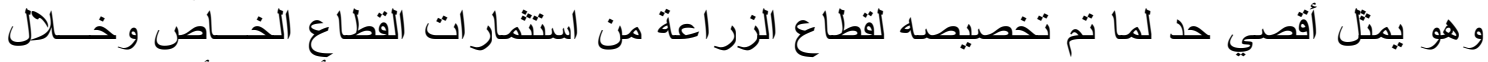

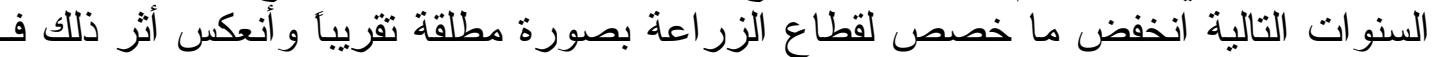

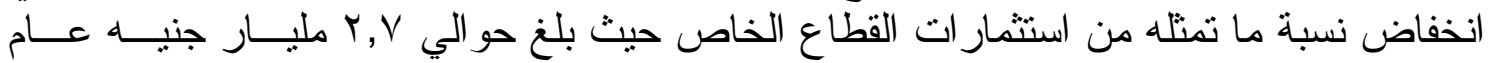

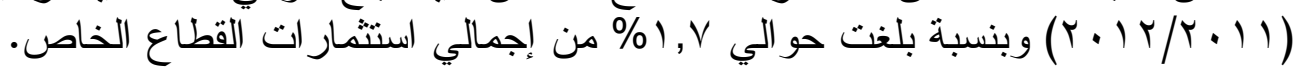

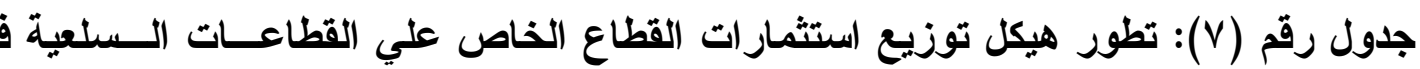

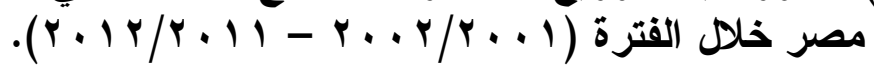

(بالأسعار الجارية وبالمليار جنيه)

\begin{tabular}{|c|c|c|c|c|c|c|c|c|c|c|c|}
\hline \multicolumn{2}{|c|}{ التشييد والبناء } & \multicolumn{2}{|c|}{ قاعهرباءع } & \multicolumn{2}{|c|}{ قطاع البترول } & \multicolumn{2}{|c|}{ قطاع الصناعة } & \multicolumn{2}{|c|}{ 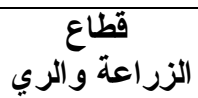 } & \multirow{2}{*}{ القطاع الخاصنار ات } & \multirow[t]{2}{*}{ السنو ات } \\
\hline$\%$ & الإجمالي & $\%$ & الإجمالي & $\%$ & الإجمالي & $\%$ & الإجمالي & $\%$ & الإجمالي & & \\
\hline$\varepsilon, \wedge$ & 1,040 & $7, \Gamma$ & $r, \cdots r$ & $1 \cdot, V$ & $r, \varepsilon \cdot \Lambda$ & $1 \cdot, \cdot$ & $r, 19$. & 11,0 & $0, \wedge 9 \wedge$ & $\Gamma_{1, \wedge \leq 7}$ & $r \ldots r / r \ldots l$ \\
\hline$\varepsilon, Y$ & $1, \leqslant Y$. & $r, \cdot$ & $\cdot, 770$ & $r T, \cdot$ & $V, V r_{0}$ & $1 \leqslant, 0$ & $\varepsilon, \wedge \vee 0$ & 9,0 & $r, 1 \wedge r$ & $r r, \tau \leq \tau$ & $r \ldots r / r \ldots r$ \\
\hline$r, r$ & $\cdot, \wedge \ldots$ & $\cdot, 0$ & $\cdot, Y \ldots$ & $\Gamma \cdot, \varepsilon$ & $11, r 71$ & $1 \cdot, 9$ & $\varepsilon, \cdot r \mu$ & $1 \cdot, \wedge$ & $\varepsilon, \ldots$ & $r v, 1 \ldots$ & $r \ldots \varepsilon / r \ldots r$ \\
\hline $1, \wedge$ & .,人ז० & - & - & rq, & $M, \cdot V_{0}$ & $I V, V$ & $\Lambda, Y \cdot \Lambda$ & 9,1 & $\varepsilon, Y_{O}$. & $\sum \nearrow, \xi \backslash \vee$ & $r \ldots 0 / T \ldots \varepsilon$ \\
\hline 0,7 & $r, V 10$ & - & - & $r V, \cdot$ & $1 \mathrm{~V}, 190$ & $1 \varepsilon, \Gamma$ & $9, \Sigma \wedge$. & $V, q$ & $0, Y \leq \varepsilon$ & TI,TYO & $r \ldots T / r \ldots 0$ \\
\hline$r_{,} \cdot$ & $r, \ldots$ & - & - & $17, \wedge$ & 17,199 & rV,r & rq, Y9o & 0,0 & $0, r O V$ & $q \vee, r \ldots$ & $r \ldots V / r \ldots r$ \\
\hline$r_{,} \cdot$ & $r, 700$ & - & - & $r), \Lambda$ & $r \wedge, Y$. & $r \wedge, r$ & M & $\varepsilon, \cdot$ & $0, Y Y T$ & $1 \% q, \cdot 1$. & $r \ldots \Lambda / r \ldots v$ \\
\hline$r, \cdot$ & $r, q \ldots$ & - & - & ro,. & & $r r, \Lambda$ & rr,VOO & $\varepsilon, r$ & $\varepsilon, 119$ & $90, \Sigma \vee 7$ & $r \ldots q / r \ldots \Lambda$ \\
\hline$Y, \varepsilon$ & $r, 1 \ldots$ & - & - & M & $\varepsilon \cdot, \Gamma ч)$ & $1 \varepsilon, \varepsilon$ & $1 \wedge, r$ & $r, \cdot$ & r,人тo & IY $Y, \vee T \wedge$ & $r \cdot 1 \cdot / r \cdot . q$ \\
\hline$r, \varepsilon$ & $\varepsilon, \wedge \ldots$ & - & - & $r T, 1$ & $r Y, V \leqslant T$ & IT,r & $\curlywedge \wedge, \wedge \wedge$. & $r, 0$ & $r, 00 \Lambda$ & $1 \leqslant 1,7 \vee 7$ & $r .11 / r \cdot 1$. \\
\hline$\cdot, 7$ & $\cdot, \wedge 9$. & - & - & $r \wedge, \cdot$ & $\varepsilon r, 0$. & $I T, V$ & $19, \Sigma V V$ & $1, V$ & $r, 791$ & IOT,OY & $r .1 T / r .11$ \\
\hline$r, q 1$ & $Y, Y \leq$ & - & - & $r \varepsilon, \wedge V$ & $Y Y, \Sigma Y$ & $1 v, 9$ & $17,0 \leq$ & 7,0 & $\varepsilon, \mu_{1}$ & $\Lambda V, Y$ & المتوسط \\
\hline
\end{tabular}

http://www.mop.gov.eg/annual.html موقع وزارة التخطيط و التعاون الدولي علي شبكة الإنترنت

أما بالنسبة لقطاع الصناعة فقد بلغ ما تم تخصيصه من استثمار ات القطاع الخــاص عـام هـام

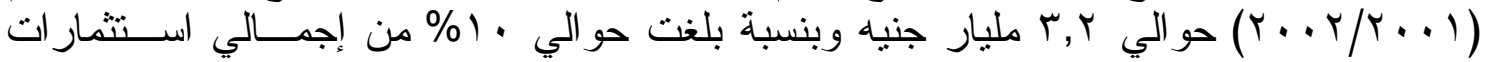

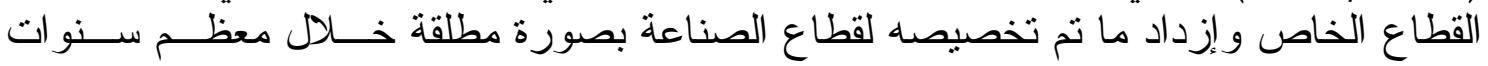

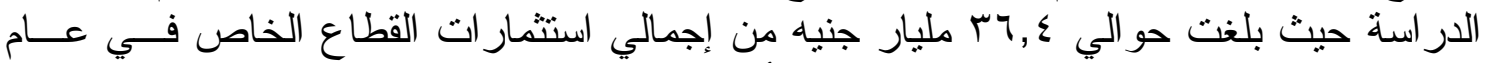

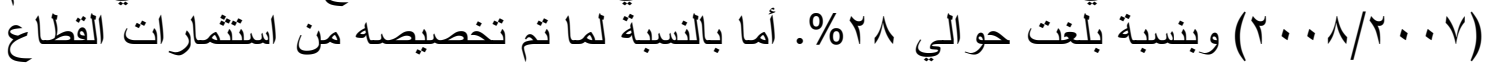

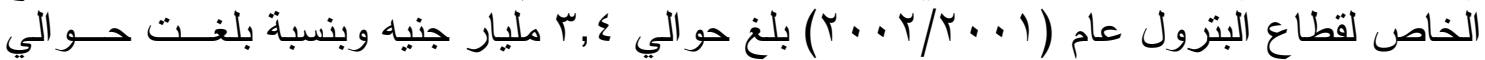

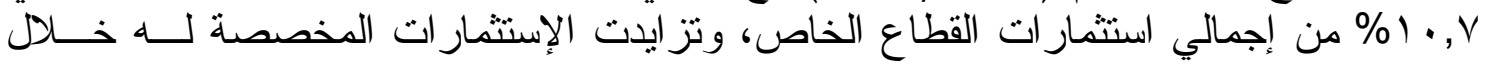

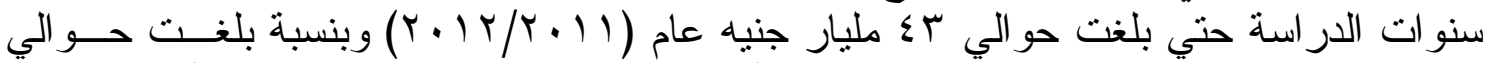

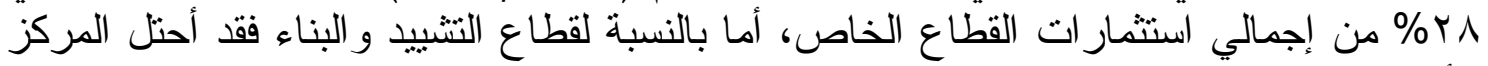

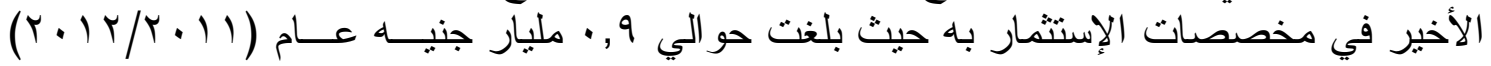

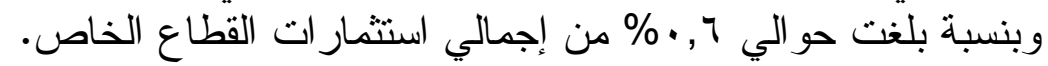

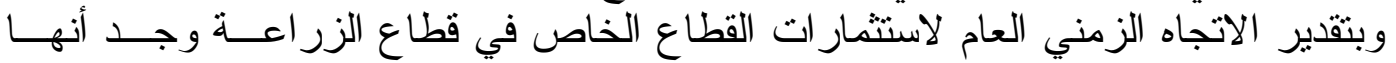

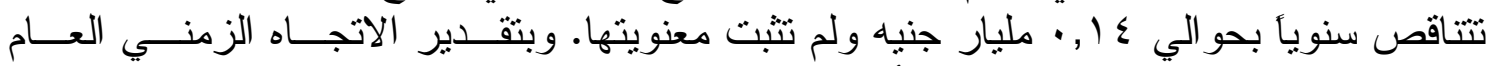

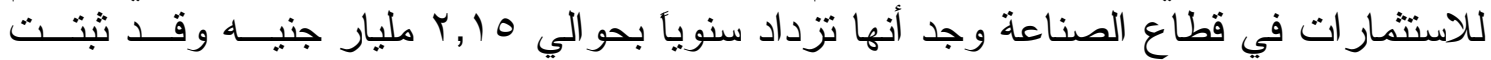


معنويتها إحصائياً. كما أن حو الي بr\% من إجمالي التغير ات الحادثة فــي قيمــة الاســثنمار ات يعكسها عنصر الزمن. وبتقدير الاتجاه الزمني العام للاستثمار ات بقطاع البترول وجد أنها تـزداد ســنوياً زيـــادة

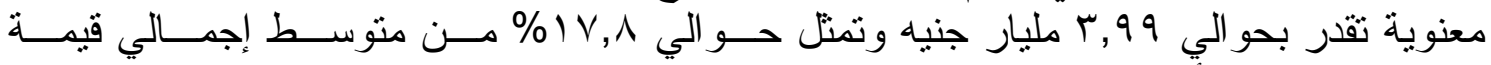

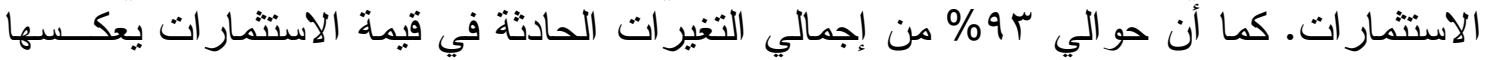

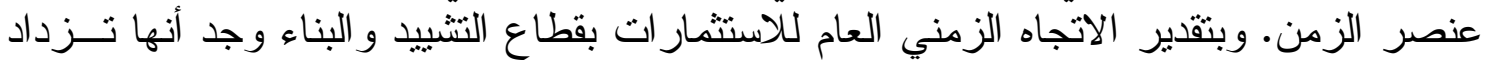

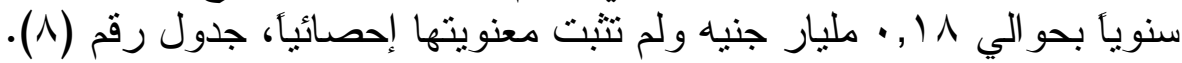

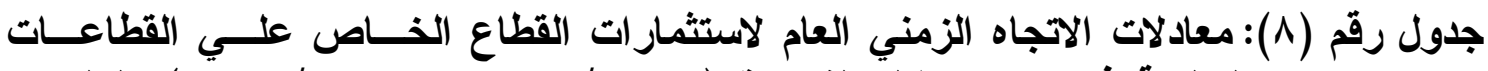

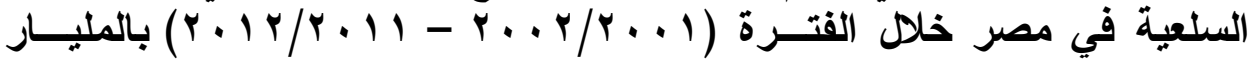

\begin{tabular}{|c|c|c|c|c|c|c|c|}
\hline 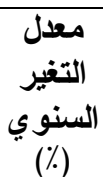 & مقار التغير (مليار جنيه) & 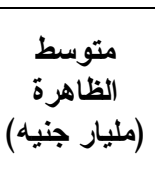 & $\mathbf{F}$ & $\mathbf{R}^{2}$ & $\mathbf{R}$ & معادلة الاتجاه الزمني العام & البيان \\
\hline$r, r o-$ & $\cdot, 1 \leq-$ & $\varepsilon, \Gamma$ & T, ז' & $\cdot, Y_{1}$ & $\cdot, \leqslant 7$ & 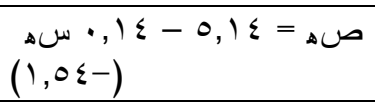 & قطاع الزر اعة \\
\hline 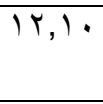 & $r, 10$ & $17,0 \leqslant$ & $* \xi, 10$ & $\cdot, \Gamma$ & $\cdot, 7$ & 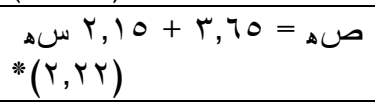 & قطاع الصناعة \\
\hline$T V, \Lambda$. & $r, 99$ & YY, \&Y & $* * 1 r \leqslant, 90$ & $\cdot, 94$ & $\cdot, 9 \mathrm{~V}$ & $\begin{array}{l}\text { صه = - } \begin{array}{l}\text { ص } r, 99+1,01 \\
* *(1), 1 \wedge)\end{array}\end{array}$ & قطاع البترول \\
\hline \multicolumn{7}{|c|}{ 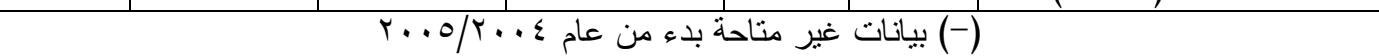 } & قطاع الكهرباء \\
\hline$\Lambda, \cdot \varepsilon$ & $\cdot, 11$ & Y,Y & r,o. & $\cdot, r T$ & $\cdot, \sum V$ & 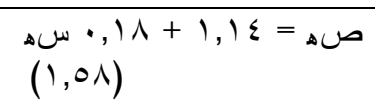 & قطاع النتشييد \\
\hline
\end{tabular}

حيث صه تثير إلي القيمة التقديرية للاستثمار بالمليار جنيه القطاع الخاص بالقطاعات السلعية في مصر

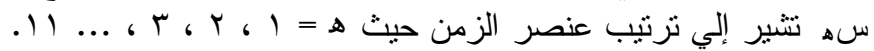

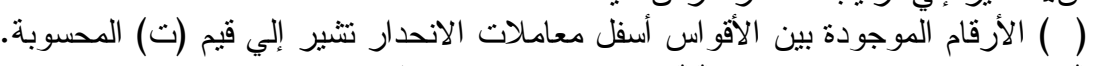

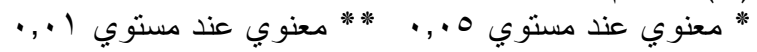

المصدر: جمعت وحسبت من البيانات الو أردة بالجدول رقدم (v).

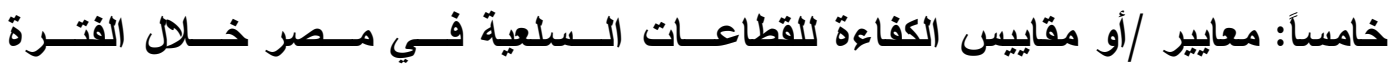
$:(r+1 r / r+11-r \ldots r / r+1)$ مفهوم الكفاءة : مفاعة

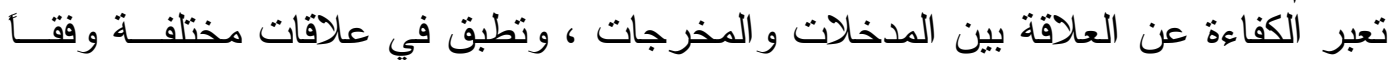

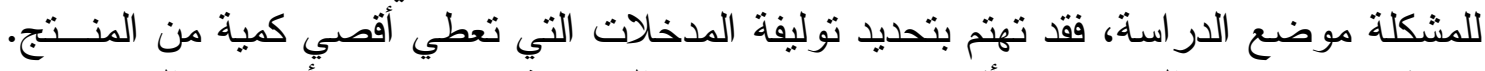

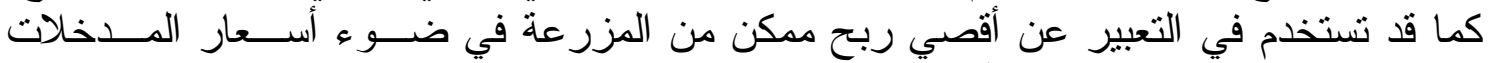

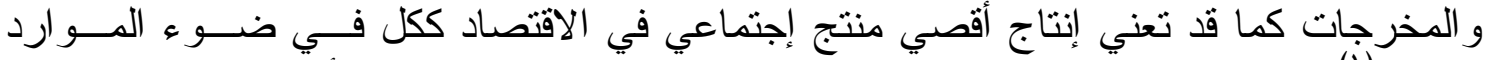

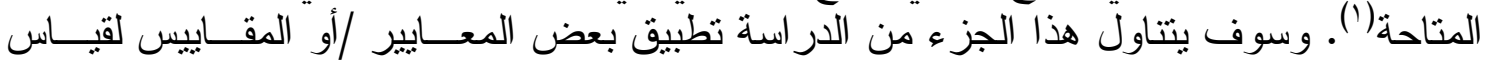

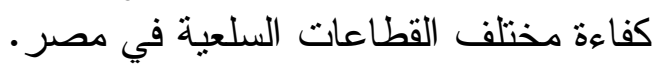

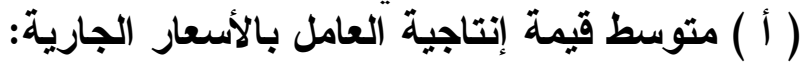

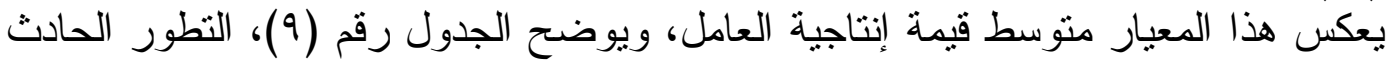

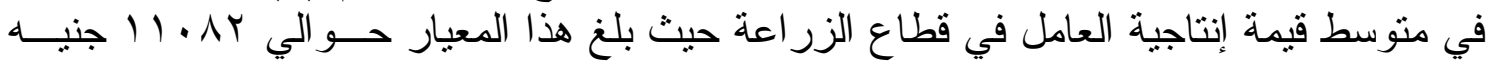

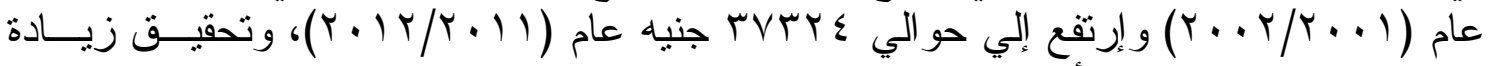

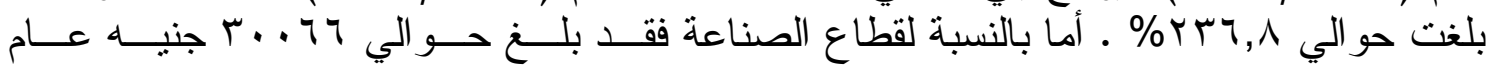

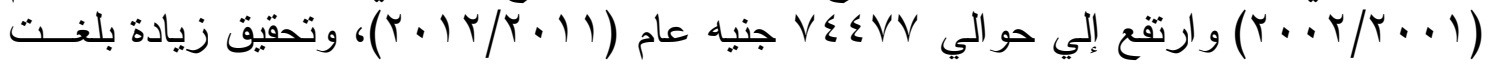




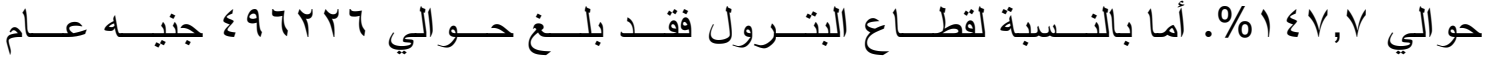

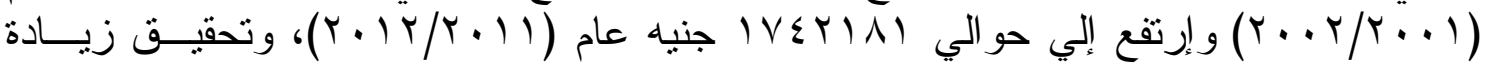

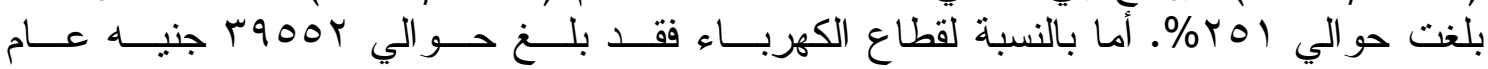

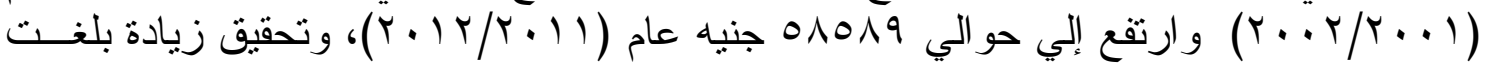

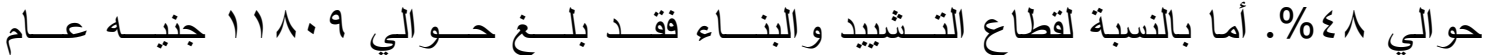

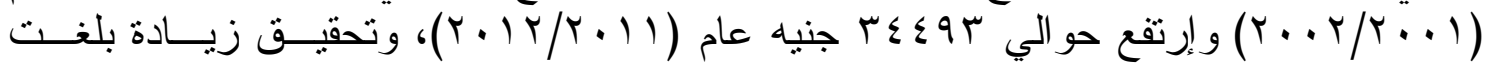

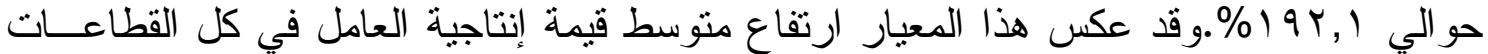

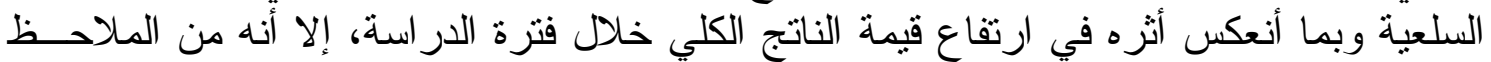

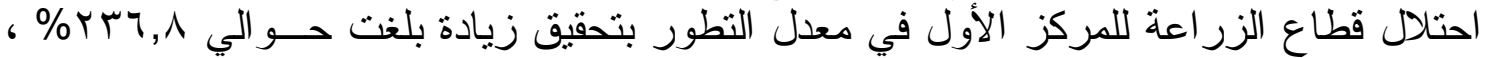

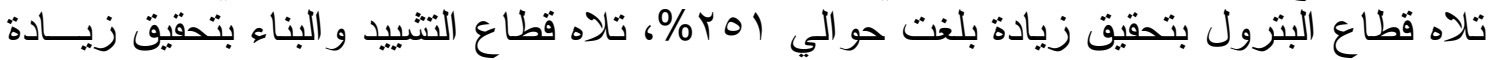

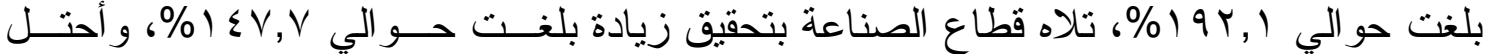

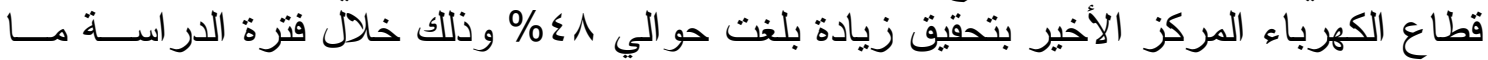

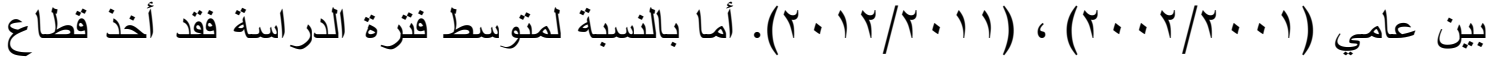

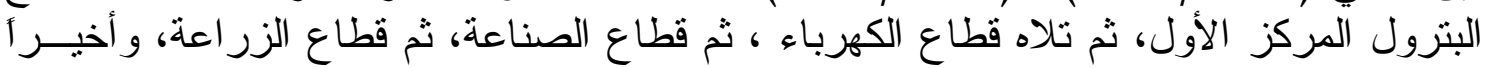

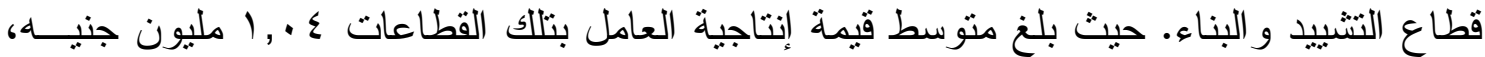

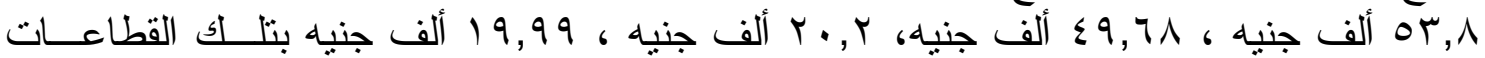
المذكورة، علي الترنيب.

(ب) معامل التوظف أو معامل رأس المال المستثمر بالنسبة للعامل:

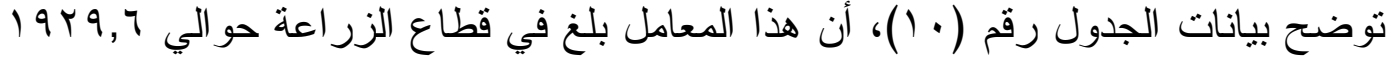

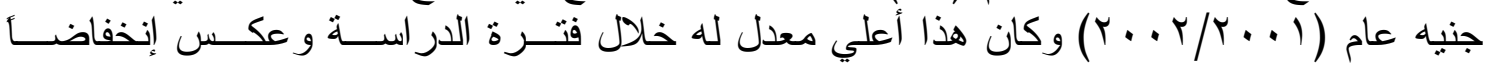

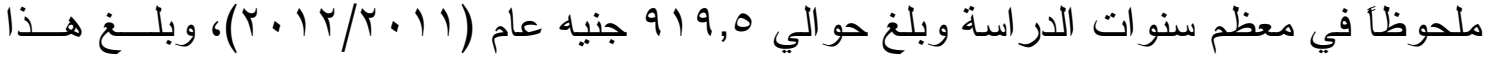

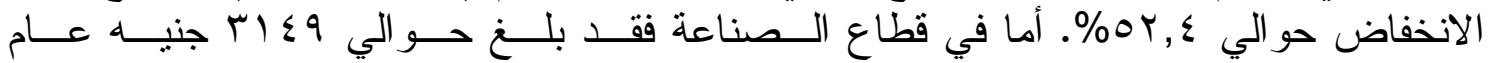

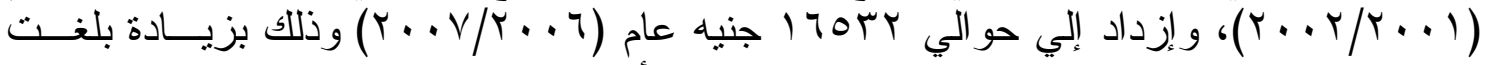

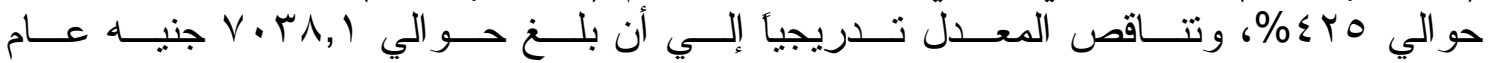

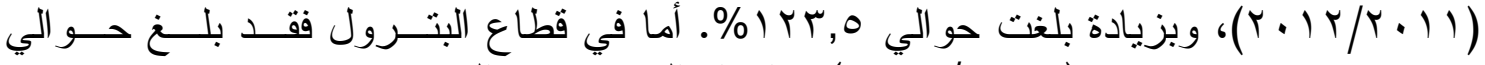

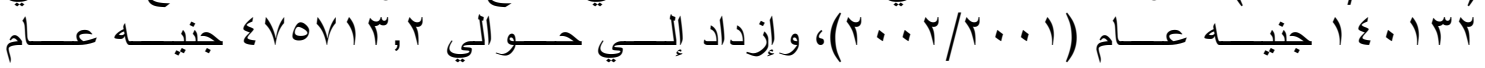

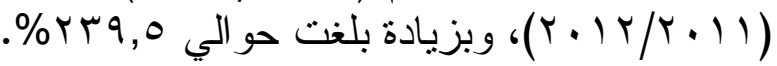

جدول رقم (9): تطور متوسط قيمة إنتاجية العامل بالقطاعات السلعية في مصر خلا بل القتهــرة . (r.11/r.1.- - r. r/r. 1)

\begin{tabular}{|c|c|c|c|c|c|}
\hline \multicolumn{5}{|c|}{ متوسط قيمة إنتاجية العامل بالجنيه ** (بالأسعار الجارية) } & \multirow{2}{*}{ السنوات } \\
\hline التثشييا و البناء & 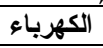 & البترول & 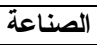 & 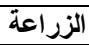 & \\
\hline 111.9 & r900r & \&97YYT & $r . .4 T$ & $11 \cdot \lambda Y$ & $r \ldots r / r \ldots 1$ \\
\hline $\mathrm{IT \cdot VT}$ & ETHAY & 079979 & TrIA. & 11741 & $r \cdot r / r \cdot r$ \\
\hline $111 \mathrm{AT}$ & $\varepsilon 0 \lambda \vee \Lambda$ & $79 . .2 \leqslant 9$ & rV.VO & 1 Tros & $r \ldots \varepsilon / r \ldots r$ \\
\hline ITAY乏 & EV9IV & AEYAVA & $r \wedge 011$ & 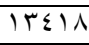 & $r \ldots o / r \ldots \varepsilon$ \\
\hline ITtry & 04.41 & 1个47.1 & $\varepsilon .04 \varepsilon$ & $1 \leq \mathrm{rAV}$ & $r \ldots T / r \ldots o$ \\
\hline 107.7 & $097 \% 1$ & 999474 & $\{011 \leqslant$ & $10 \mathrm{THN}$ & $r \ldots v / r \ldots r$ \\
\hline $1911 \leqslant$ & 70141 & ITTYYQA & OTrar & T.AYY & $r \cdots \Lambda / r \cdots v$ \\
\hline rדAYq & ATrTY & ITYMIE. & $7.8 \cdot 1$ & $r \leqslant \leqslant \pi$ & $r \ldots q / r \cdots \Lambda$ \\
\hline 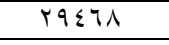 & $\varepsilon 0 \wedge \varepsilon Y$ & Irqsor| & $T \sum V 1$. & rAY90 & $r \cdot 1 \cdot / r \ldots q$ \\
\hline$r+10$ & $\varepsilon 9 \% \cdot \varepsilon$ & $1 \leq \varepsilon \varepsilon \varepsilon \varepsilon \varepsilon$ & $797 \mathrm{VV}$ & rYAEQ & $r \cdot 11 / r \cdot 1$. \\
\hline$r \leqslant \varepsilon 9 r$ & 01019 & $\mid V \leqslant$ VIAI & $V \Sigma \varepsilon V Y$ & TVYTY & ${ }^{*} Y \cdot \mid r / r+11$ \\
\hline 19911 & orArq & $1 .+7 T V 1$ & $\sum 97 \vee 7$ & r.19 & المتوسط \\
\hline
\end{tabular}

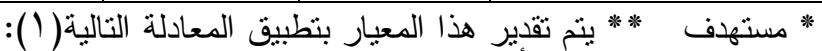
قيمة الإنتاج الكلي بالأسعار الجارية في سنة ما في قطاع مالـا ، ، ارتفاع قيمة المعيار يعني زيادة الكفاءة، نظر آلأن معدل

(1) راجع في ذللك: أحمد محمود عبد العزيز ، إنجاز ات ومعوقات التتمية الاقتصادية الزر اعية في مصر ، رسالة ماجستير ،

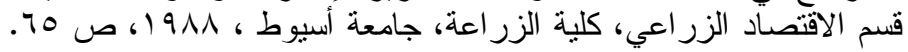


عدد العمال في نفس السنة لهذا القطاع

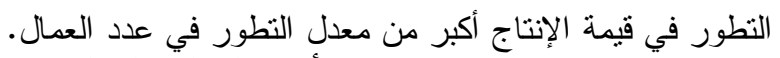

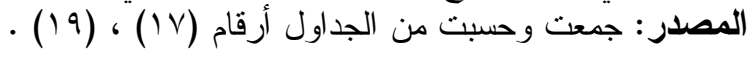

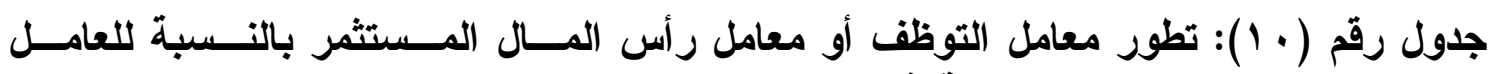

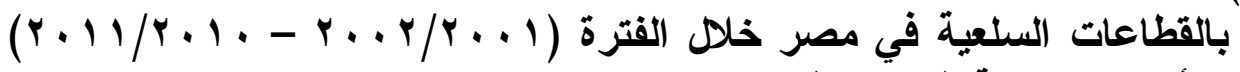

بالأسعار الجارية ( بالجنيه).

\begin{tabular}{|c|c|c|c|c|c|}
\hline \multicolumn{5}{|c|}{ معامل التوظف * * } & \multirow{2}{*}{ السنوات } \\
\hline التشييا و البناء & الكهرباء & البترول & الصناعة & الزر اعة & \\
\hline $1 \leqslant r V, 0$ & Tו & $1 \varepsilon .1 T r, 1$ & ए) 19,1 & $19 r 9,7$ & $r \ldots r / r \ldots l$ \\
\hline $1 \% \wedge 7,9$ & rAVO.,. & $10 \leqslant \vee 07, V$ & $r \varepsilon \cdot r, \Lambda$ & IYVY,Y & $r \ldots r / r \ldots r$ \\
\hline$\vee \vee 0,1$ & OYтA1,... & $r \cdot V \leqslant r \Lambda, \Lambda$ & YษA1,9 & $1 \leqslant \wedge \neg, \wedge$ & $r \ldots \varepsilon / r \ldots r$ \\
\hline$\Lambda Y V, \Sigma$ & $00 Y 10, r$ & $1 \vee 90 \vee \varepsilon, \cdot$ & $0 \leqslant \leqslant 0, Y$ & $1 \leqslant r \wedge, \Lambda$ & $r \ldots 0 / r \ldots \varepsilon$ \\
\hline$r \wedge \cdot \varepsilon, 1$ & $0 \leqslant .9 \leqslant,$. & $r \leqslant Y Y \mid 0, T$ & $097 \cdot, 0$ & 1047,1 & $r \ldots T / r \ldots o$ \\
\hline $1 T Y \xi, Y$ & $011 \leqslant V, V$ & r & 170rY, & $1 \leq 7 \cdot, 9$ & $r \ldots v / r \ldots r$ \\
\hline$r \cdot 9 \wedge, 1$ & $V .009, r$ & $r \leqslant \Lambda \backslash \backslash, r$ & $1090 \cdot, r$ & $1 \leqslant \wedge \vee, \cdot$ & $r \ldots r / r \ldots v$ \\
\hline rrrq,7 & $997 \leqslant 1,1$ & M1070r,A & $1 \cdot 7 \cdot \varepsilon, V$ & $1 Y r V, 0$ & $r \ldots q / r \ldots 1$ \\
\hline TMTY,Y & $\varepsilon \wedge \Lambda \cdot q, r$ & $\Sigma \vee \wedge \wedge \wedge \uparrow, \wedge$ & NOr., V & $11 \wedge 0,1$ & $r \cdot 1 \cdot / r \cdot . q$ \\
\hline$\Gamma \cdot \Lambda 1, r$ & $\leqslant 99 \leq \cdot, \wedge$ & rTIT,r,V & V09T,r & 1111,0 & $r .11 / r \cdot 1$. \\
\hline 179,0 & $07 \leqslant \leqslant \leqslant, \Lambda$ & $\varepsilon V O V I T, Y$ & $V \cdot r \wedge, I$ & 919,0 & $* r \cdot 1 r / r \cdot 11$ \\
\hline$I \vee \vee \Lambda, \vee$ & $0 \leqslant \wedge \neg \cdot, r$ & 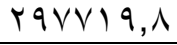 & $\vee \wedge 9 \wedge, 1$ & $1 \% \vee 7, r$ & المتو سط \\
\hline
\end{tabular}

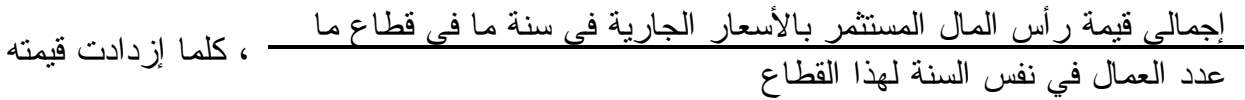

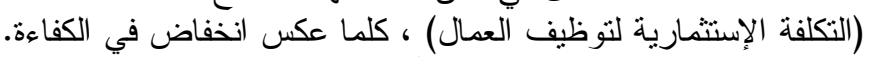

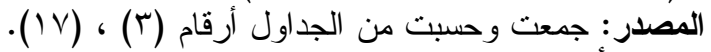

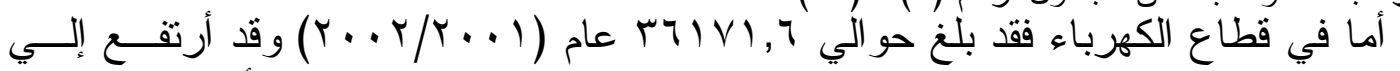

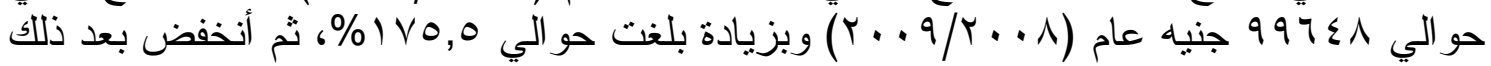

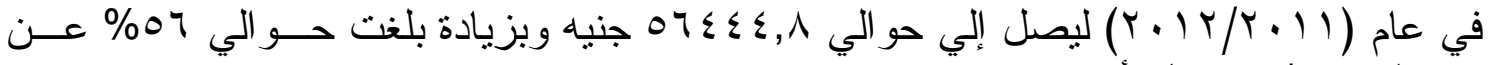

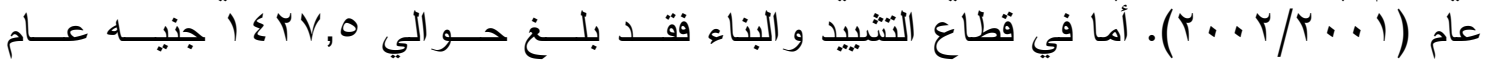

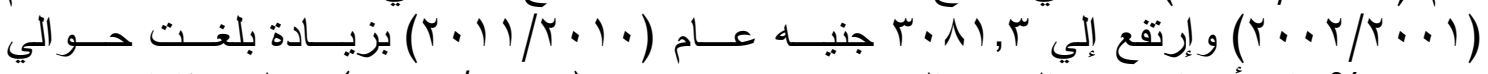

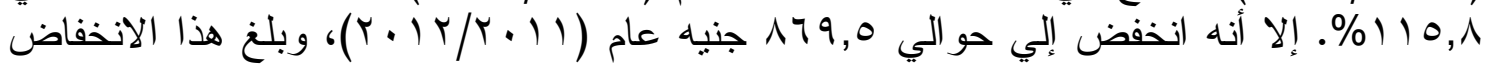

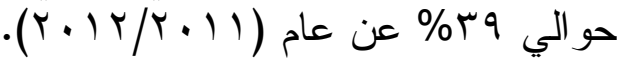

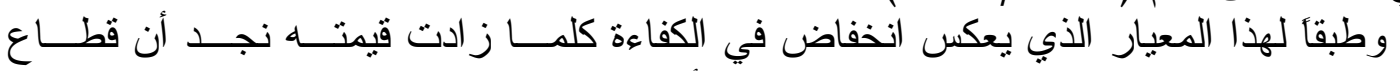

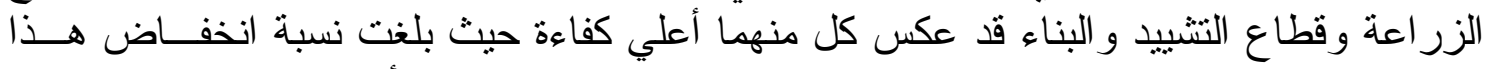

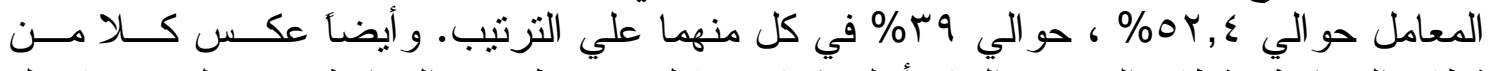

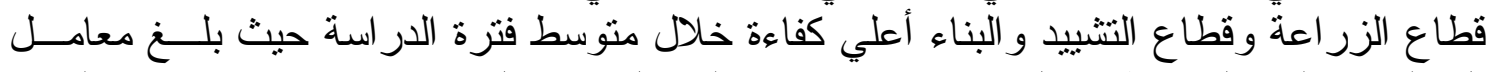

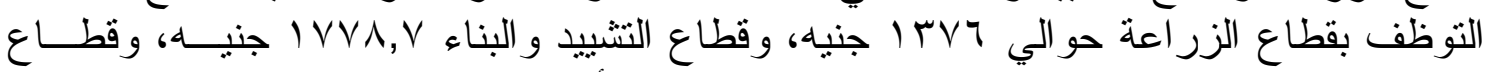

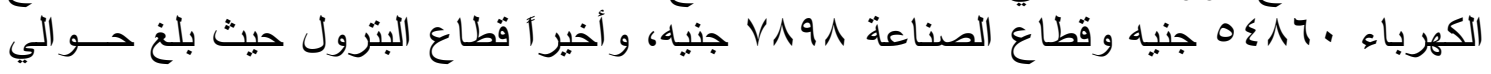

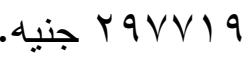

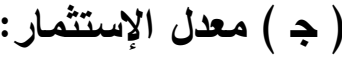

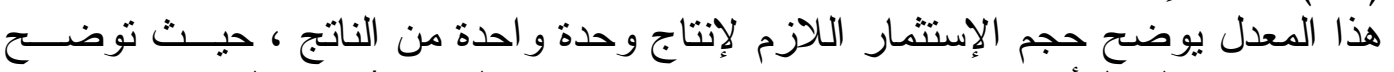

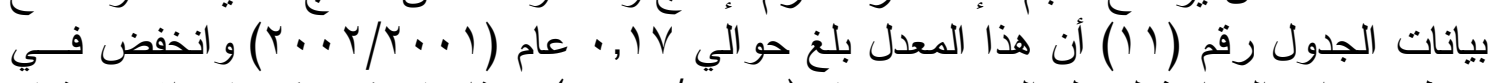

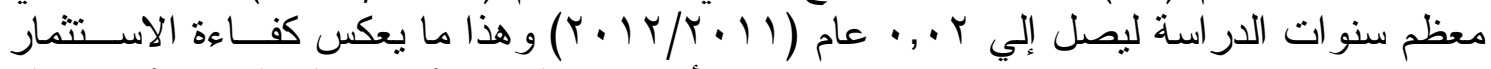

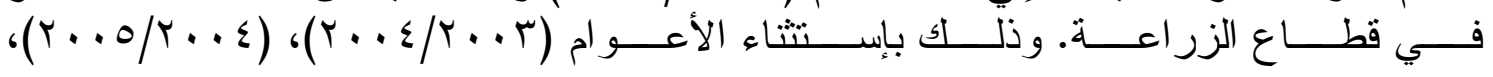

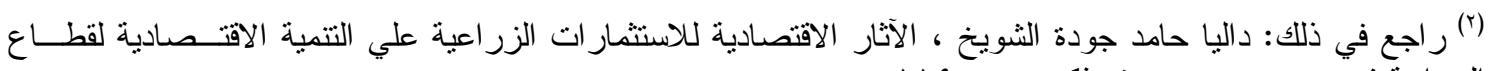

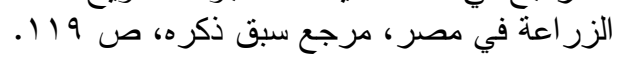




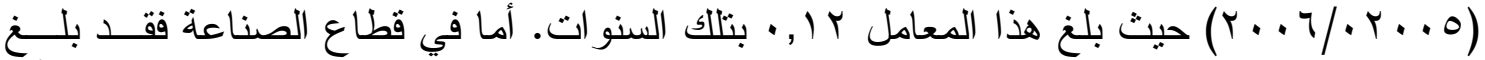

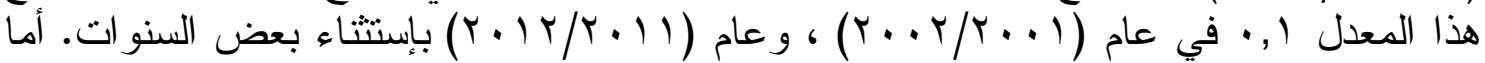

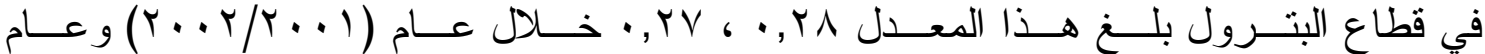

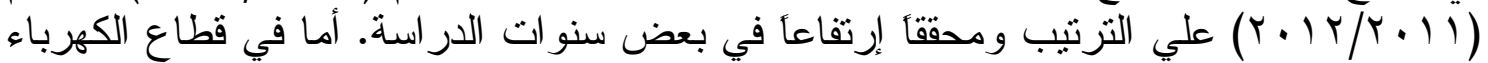

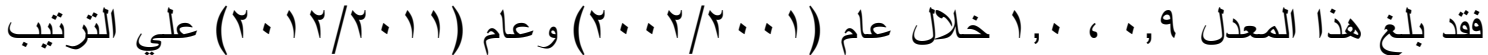

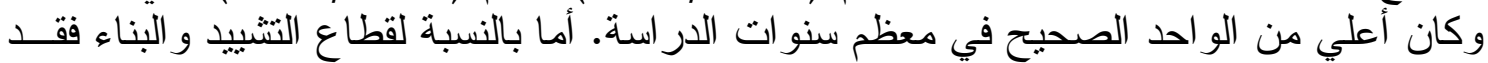

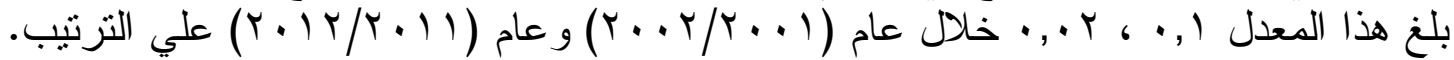

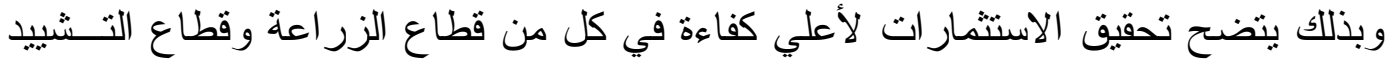

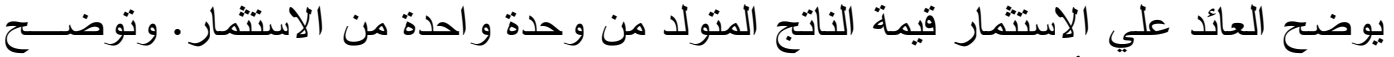

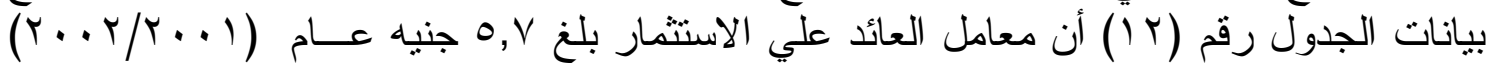

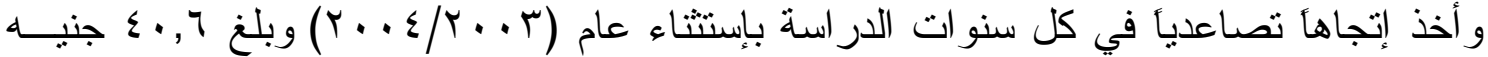

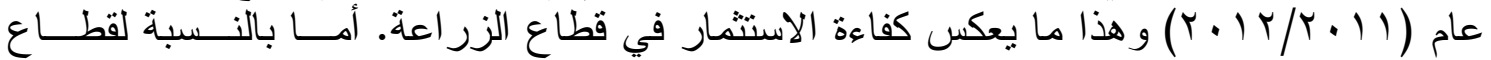

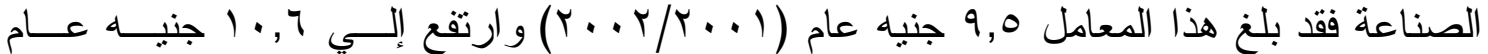

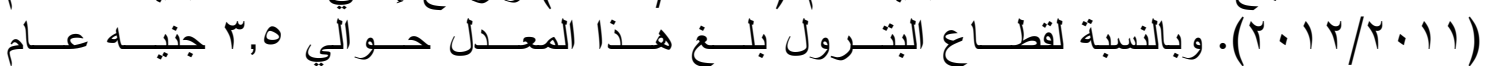

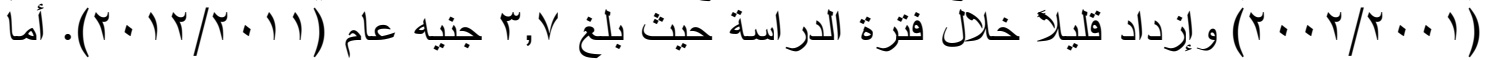

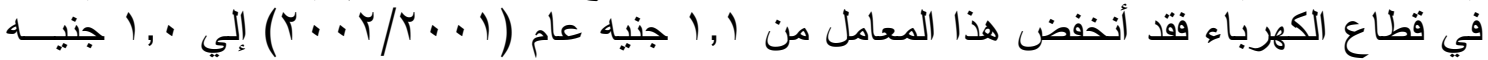

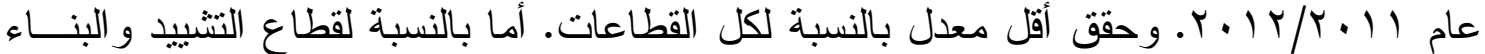

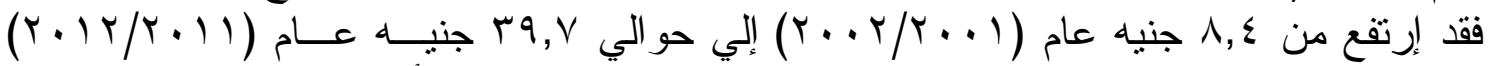

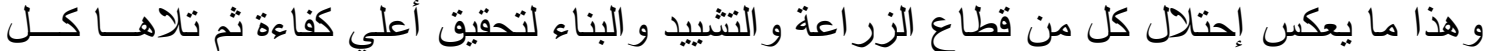

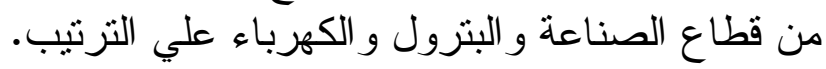

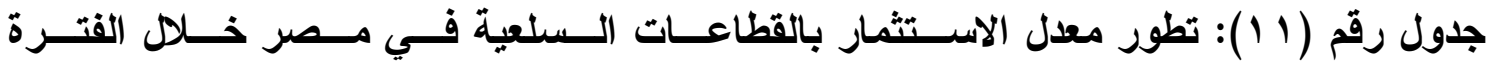

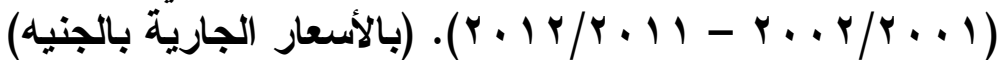

\begin{tabular}{|c|c|c|c|c|c|}
\hline \multicolumn{5}{|c|}{ معدل الاستثمار } & \multirow{2}{*}{ السنوات } \\
\hline قطاع التشيبد و البناء & قطاع الكهرباء & قطاع البترول & قطاع الصناعة & قطاع الزراعة & \\
\hline$\cdot, 1$. & $\cdot, 9$ & $\cdot, Y \wedge$ &., 1 & $\cdot, 1 \mathrm{~V}$ & $r \ldots r / r \ldots 1$ \\
\hline$\cdot, 1 Y$ & $\cdot, \mathrm{V}$ & $\cdot, \mathrm{r} \Lambda$ & $\cdot, 1$ & $\cdot, 11$ & $r \ldots r / r \ldots r$ \\
\hline$\cdot, 1$ & 1,1 & $\bullet, \Gamma$ & $\cdot, 1$ & $\cdot, 1 Y$ & $r \ldots \varepsilon / r \ldots r$ \\
\hline$\cdot, 1$ & $1, \varepsilon$ & $\cdot, Y_{1}$ & $\cdot, 1 \leq$ & $\cdot, 1 Y$ & $r \ldots o / r \ldots \varepsilon$ \\
\hline$\cdot, Y$ & $1, \cdot$ & $\cdot, \leqslant 1$ & $\cdot, 10$ & $\cdot, 1 Y$ & $r \ldots r / r \ldots o$ \\
\hline$\cdot, 1$ & $\cdot, \wedge$ & $\cdot, r$ & $\cdot, r V$ & $\cdot, 1 \cdot$ & $r \ldots v / r \ldots r$ \\
\hline$\cdot, 1 Y$ & 1,1 & $\cdot, r V$ & $\cdot, \Gamma$ & $\cdot, 1$ & $r \ldots \lambda / r \ldots v$ \\
\hline$\cdot, 1$. & $1, r$ & $\cdot, Y T$ & $\cdot, 11$ &., .0 & $r \ldots q / r \ldots 1$ \\
\hline$\cdot, 1$ & 1,1 & $\cdot, r V$ & $\cdot, 1 \pi$ & $\cdot, \cdot \varepsilon$ & $r \cdot 1 \cdot / r \ldots q$ \\
\hline$\cdot, 1$ & $1, \cdot$ & $\cdot, r \mu$ & $\cdot, 11$ & $\cdot, \cdot r$ & $r+11 / r \cdot 1$. \\
\hline$\cdot, \cdot r$ & $1, \cdot$ & $\cdot, r V$ &., 1 . & $\cdot, \cdot r$ & $r \cdot 1 r / r \cdot 11$ \\
\hline$\cdot, \cdot 1$ & 1,1 & $\cdot, r q$ & $\cdot, 17$ & $\cdot, .9$ & المتوسط \\
\hline
\end{tabular}

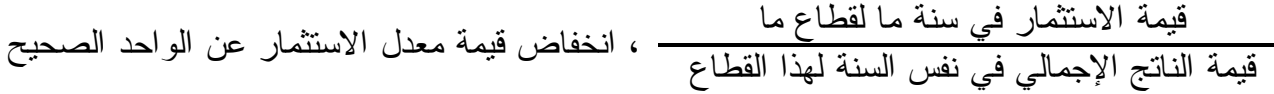

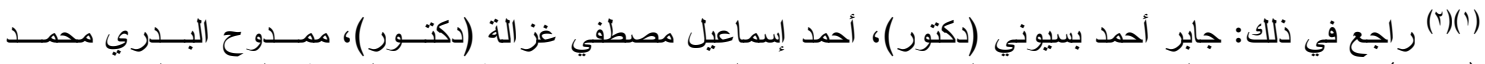

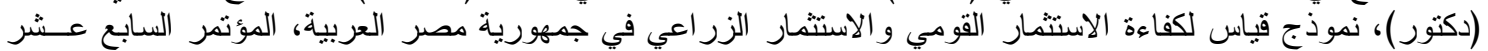

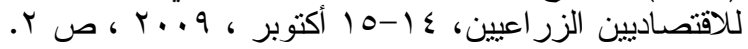


بعكس كفاءة في الاستثمار الزر اعي و العكس عند زيادة هذا المعدل.

المصدر : جمعت وحسبت من الجداول أرقام (r) ، (19) (1)

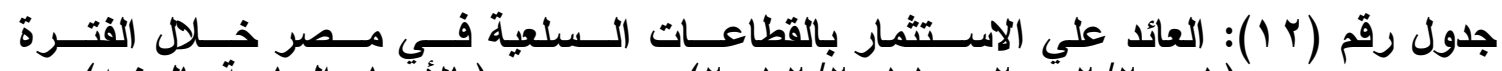

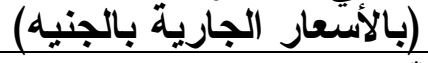
. $(r+r / r+11-r \cdot r / r+1)$

\begin{tabular}{|c|c|c|c|c|c|}
\hline \multicolumn{5}{|c|}{ العائد علي الاستثمار * } & \multirow[b]{2}{*}{ السنوات } \\
\hline قطاع التشييا و البناء & قطاع الكهرياء & قطاع البترول & قطاع الصناعة & قطاع الزراعة & \\
\hline$\Lambda, r$ & 1,1 & $r, 0$ & 9,0 & $0, \mathrm{~V}$ & $r \ldots r / r \ldots l$ \\
\hline$\Lambda, V$ & 1,0 & $r, \tau$ & $9, v$ & 9,1 & $r \ldots r / r \ldots r$ \\
\hline $10, Y$ & $\cdot, 9$ & $r, Y$ & $1 \mu, \Lambda$ & $\Lambda, \xi$ & $r \ldots \varepsilon / r \ldots r$ \\
\hline $10, \xi$ & $\cdot, 9$ & $\varepsilon, 9$ & $V, 1$ & $9, \Gamma$ & $r \ldots o / r \ldots \varepsilon$ \\
\hline$\varepsilon, \Lambda$ & 1,0 & 0, & 7,1 & $9, \xi$ & $r \ldots \tau / r \ldots 0$ \\
\hline 9,7 & $1, Y$ & $r, r$ & $Y, V$ & $1 \cdot, 0$ & $r \ldots V / r \ldots T$ \\
\hline 9,1 & $\cdot, 9$ & $\Gamma, \tau$ & $\mu, \mu$ & $1 \varepsilon$, & $r \ldots \wedge / r \ldots v$ \\
\hline 11,0 & $\cdot, \wedge$ & $r, q$ & 0,0 & $19, V$ & $r \ldots q / r \ldots 1$ \\
\hline$I^{1}, 7$ & $\cdot, 9$ & $T, V$ & $V, 7$ & rT, q & $r \cdot 1 \cdot / r \ldots q$ \\
\hline$|\cdot, \varepsilon|$ & $1, \cdot$ & $\varepsilon, \Gamma$ & 9,1 & $T V, \Lambda$ & $r .11 / r .1$. \\
\hline$r q, v$ & $1, \cdot$ & $\Gamma, \mathrm{r}$ & $1 \cdot, 7$ & $\varepsilon \cdot, 7$ & $r .1 T / r .11$ \\
\hline$I T, Y$ & $1, \cdot$ & $r, \Lambda$ & $\vee, \Lambda$ & $17, Y$ & المتوسط \\
\hline
\end{tabular}

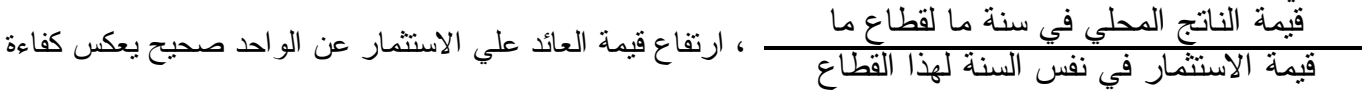

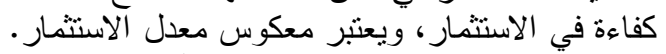

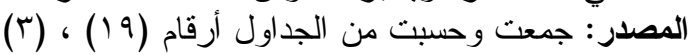

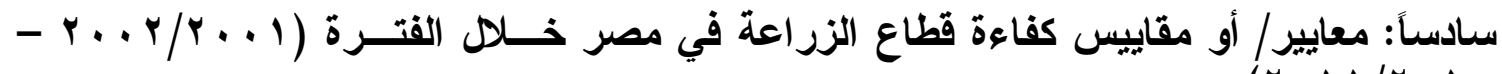

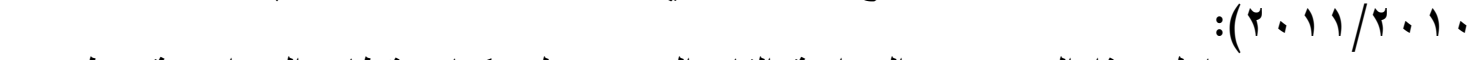
سوف يتتاول هذا الجزء من الار اسة إلقاء الضوء عـ علي كفاءة قطاع الزر اعــة بتطبيـق

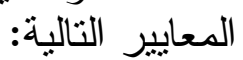

( أ ) متوسط إنتاجية العامل بالقيمة المضافة بالأسعار الجارية:

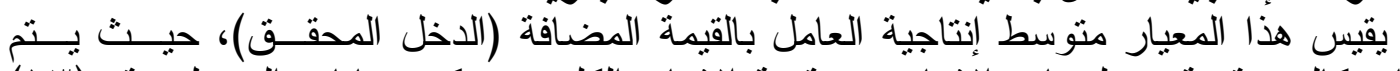

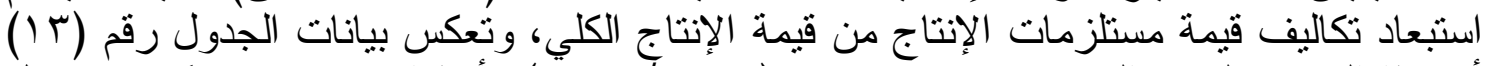

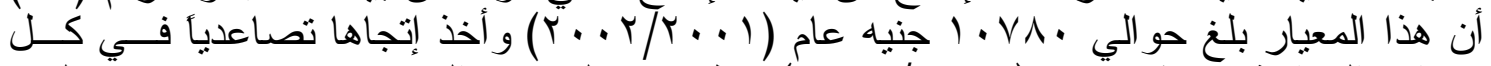

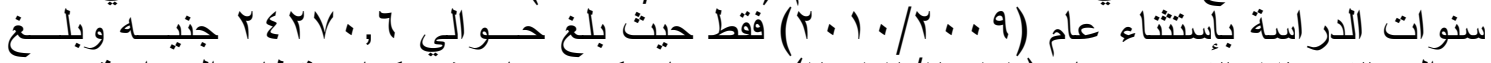

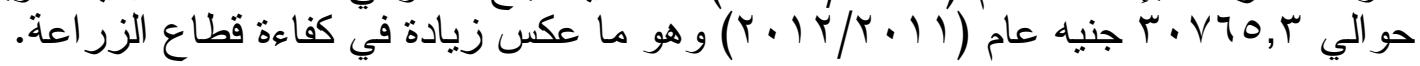

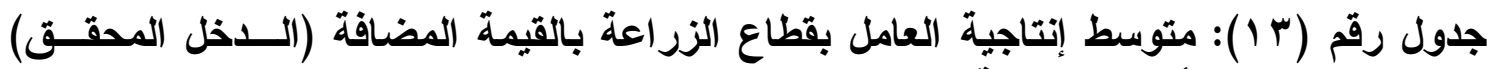

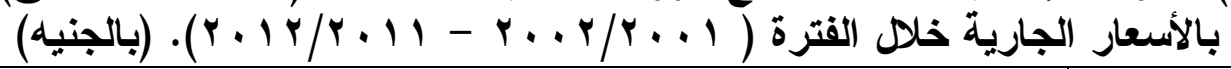

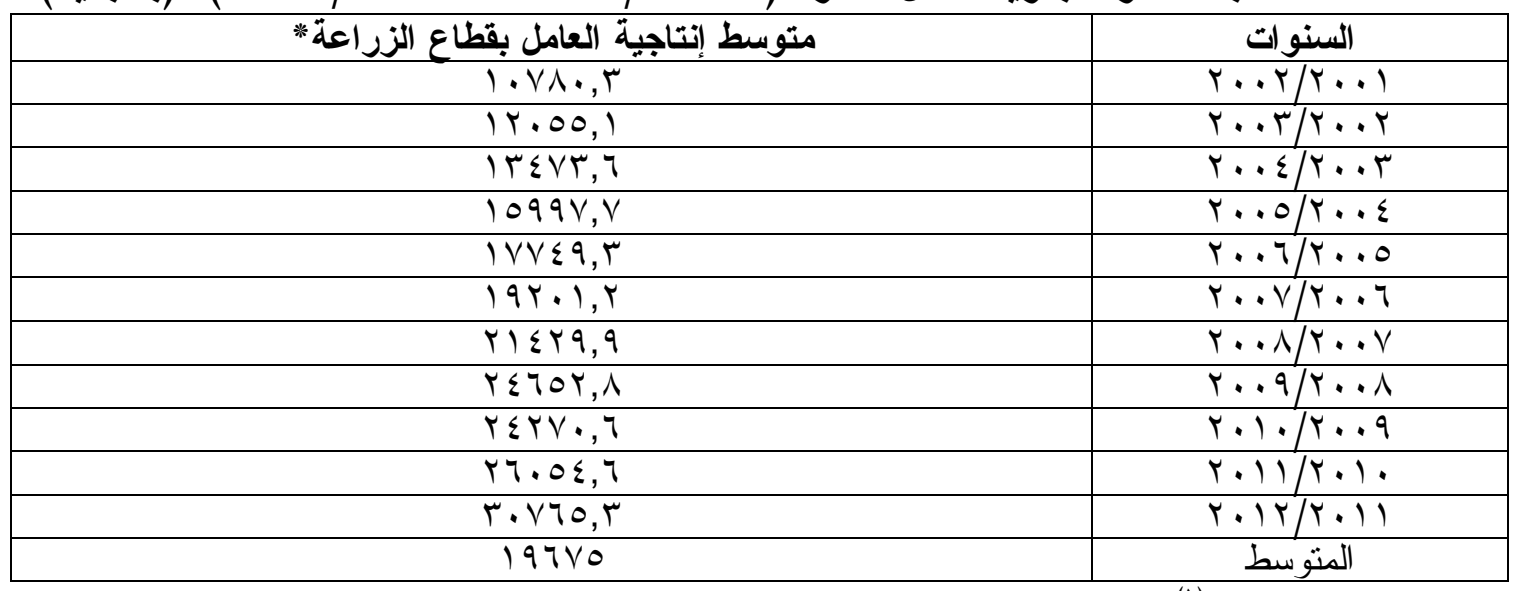

= (') متوسط إنتاجية العامل

(1) راجع في ذلك : أحمد عبد العزيز، إنجاز ات ومعوقات التتمية الاقتصادية الزر اعية في مصر، مرجع سبق ذكره ، ص 10. 


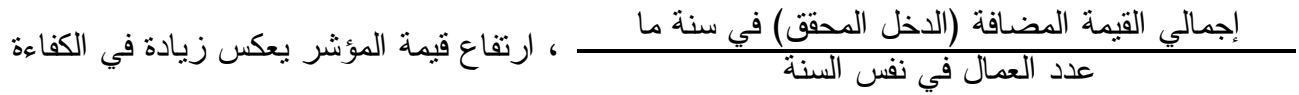

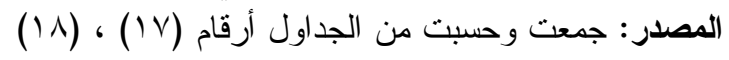

\section{( ب ) معامل إنتاجية وحدة مستلزمات الإنتاج بالأسعار الجارية:}

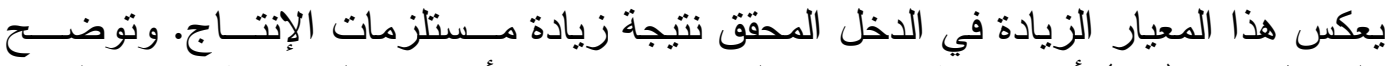

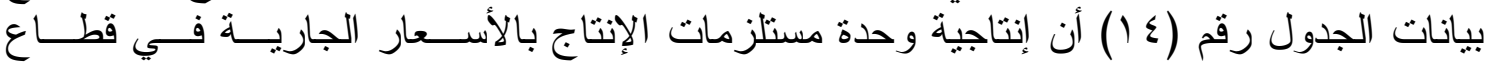

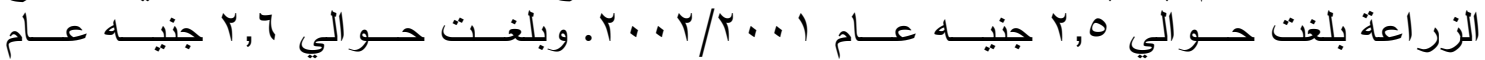

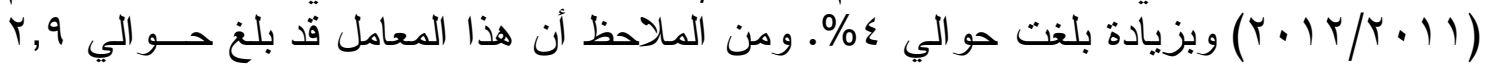

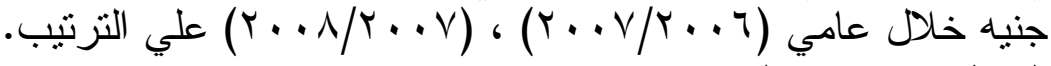

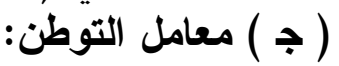

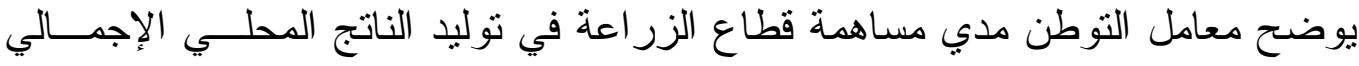

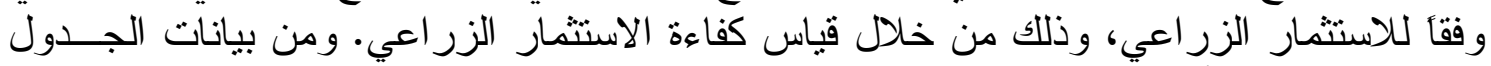

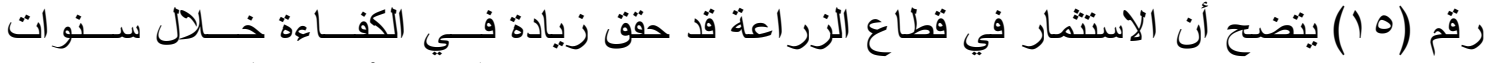

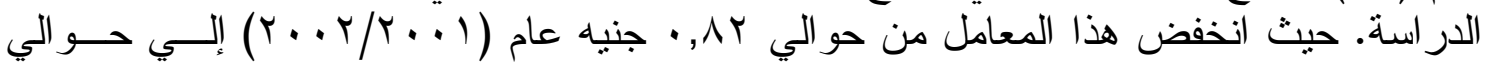

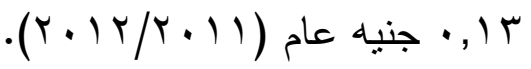

جدول رقم (ع 1): تطور إنتاجية وحدة مستلزمات الإتتاج بقطاع الزراعة في مصر خلا الم الفترة

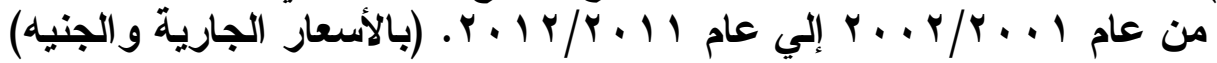

\begin{tabular}{|c|c|c|c|}
\hline 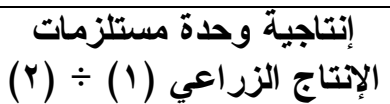 & قالإنتاج (Y (Y) & الزرافي الاخل (1) & السنوات \\
\hline$r, 0$ & $r, 1$ & or, 7 & $r \ldots r / r \ldots l$ \\
\hline$r, 0$ & Tr,A & $7 ., 0$ & $r \ldots r / r \ldots r$ \\
\hline Y, $\Sigma$ & $r \wedge, r$ & $7 \Lambda, 0$ & $r \ldots \Sigma / r \ldots r$ \\
\hline$\overline{r, \Lambda}$ & $r q, r$ & $\Lambda Y, 0$ & $\gamma_{\ldots} .0 / \gamma \ldots \varepsilon$ \\
\hline Y,V & $r \varepsilon, 1$ & $9 r, 9$ & $r \ldots T / r \ldots o$ \\
\hline$r, q$ & ro,. & T. Y, $\varepsilon$ & $r \ldots v / r \ldots T$ \\
\hline$r, q$ & $r q, 7$ & $117, r$ & $r \ldots \Lambda / r \ldots V$ \\
\hline$r, \Lambda$ & $\sum \wedge, 9$ & $1 \mu 4, V$ & $r \ldots q / r \ldots \Lambda$ \\
\hline$r, V$ & 01,5 & $1 \% \Lambda, 1$ & $r \cdot 1 \cdot / r \ldots q$ \\
\hline$r, 7$ & $0 \wedge, 7$ & $10 \cdot, \mathrm{V}$ & $r \cdot 11 / r \cdot 1 \cdot$ \\
\hline$r, T$ & $V \cdot, r$ & $1 \vee 9, V$ & $r \cdot 1 r / r \cdot 11$ \\
\hline$\overline{Y, V}$ & $\varepsilon \cdot, \cdot$ & $1 \cdot V, \varepsilon$ & المتوسط \\
\hline
\end{tabular}

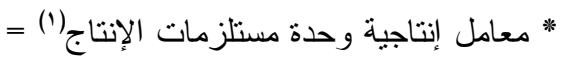

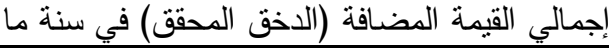

إجمالي قيمة مستلزمات الإنتاج بالأسعار الجارية في نفس السنة فالسنة ارتفاع قيمة هذا المعامل يعكس زيادة في الكفاءة.

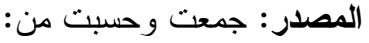

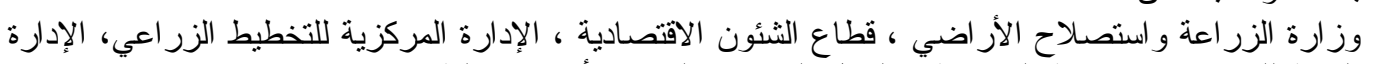

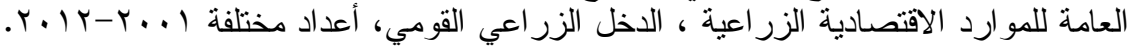




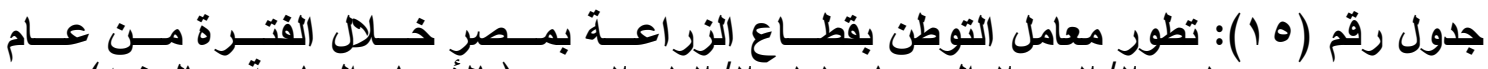

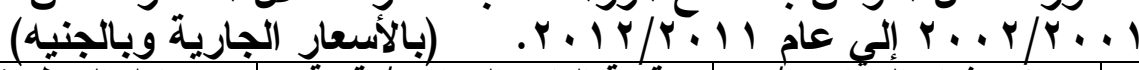

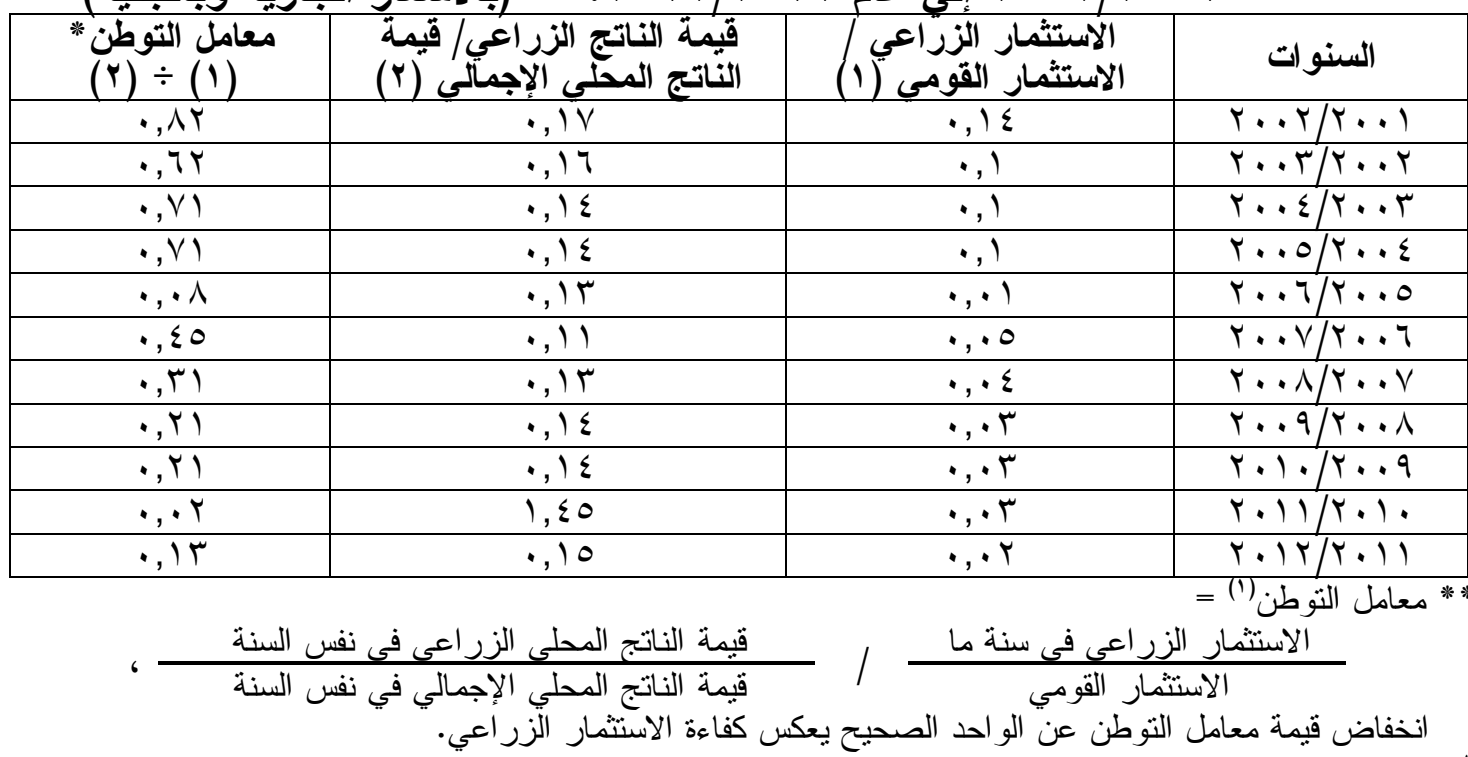

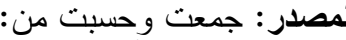

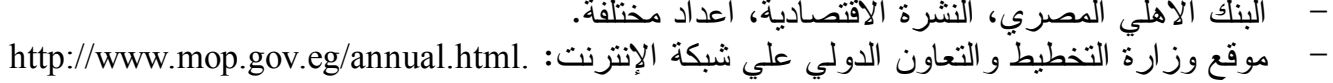

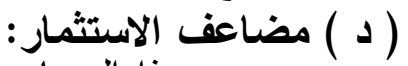

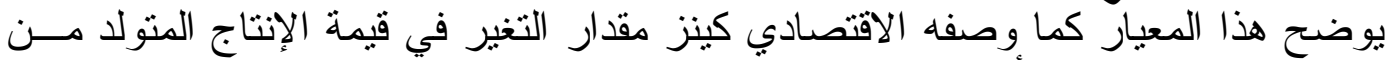

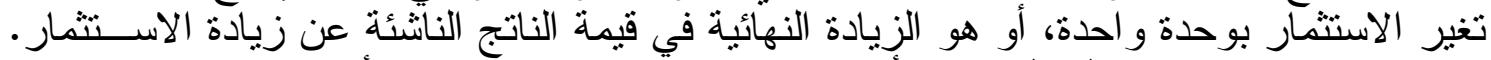

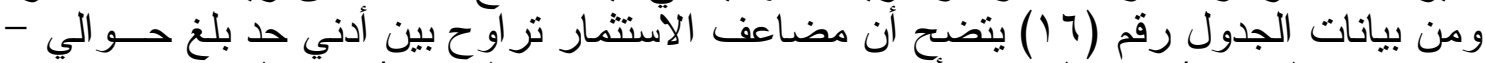

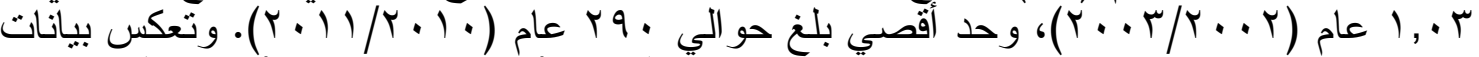

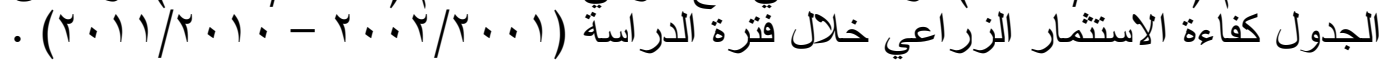

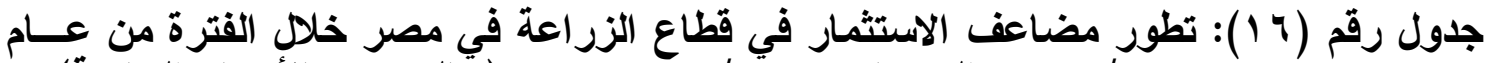

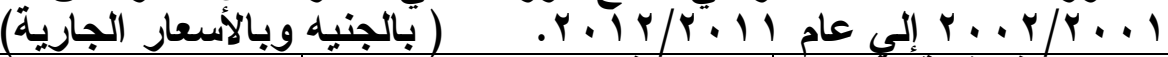

\begin{tabular}{|c|c|c|c|}
\hline مضاعف الاستثثـار * (1) & القومي في الاستثمار الزراعيه) (r) & 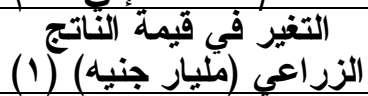 & السنوات \\
\hline$\cdot, Y \wedge$ & $1, \varepsilon+$ & $\cdot, \varepsilon$ & $r \ldots r / r \ldots l$ \\
\hline $1, \cdot r-$ & $r, Y-$ & $r, r$ & $r \ldots r / r \ldots r$ \\
\hline$\varepsilon, 0$. & $1, Y+$ & $0, \varepsilon$ & $r \ldots \varepsilon / r \ldots r$ \\
\hline $0 \leqslant, \ldots$ & $\cdot, 1-$ & $0, \xi$ & $r \ldots .0 / \mu_{\ldots} \ldots$ \\
\hline$T \cdot, Y$. & $\cdot, 7 \cdot+$ & 7,1 & $r \ldots r / r \ldots 0$ \\
\hline MY,O.- & $\cdot, Y-$ & 7,0 & $r \ldots V / r \ldots T$ \\
\hline $1 \cdot \varepsilon, \ldots$ & $\cdot, r+$ & $r, r$ & $r \ldots \Lambda / r \ldots V$ \\
\hline $1 \wedge, \vee 0-$ & $1, Y-$ & YY,O & $r \ldots q / r \ldots 1$ \\
\hline $1 Y V, 9 \cdot-$ & $\cdot, Y-$ & $r 0,0$ & $r \cdot 1 \cdot / r \ldots q$ \\
\hline$r q \cdot, \cdots$ & $\cdot, 1+$ & rq,. & $r .11 / r \cdot 1$. \\
\hline $1 \wedge, \vee \cdot-$ & $1,0-$ & $r \Lambda, \cdot$ & $r .1 T / r \cdot 11$ \\
\hline
\end{tabular}

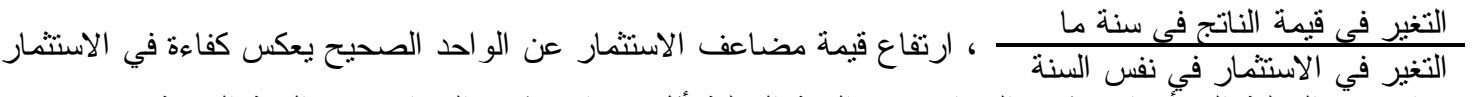

*** تثير الإثارة السالبة إلي أن الاستثمار الزراعي في السنة الحالية أقل من الاستثمار الزر اعي في السنة السابقة.

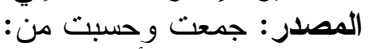

- البنك الأهلي المصري، النشرة الاقتصادية، أعداد مختلفة.

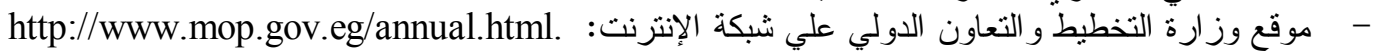

(†) راجع في ذلك : جابر أحمد بسيوني (دكتور)، و آخرون، نموذج قياس لكفاءة الاستثمار القومي و الاستثمار الزر اعي

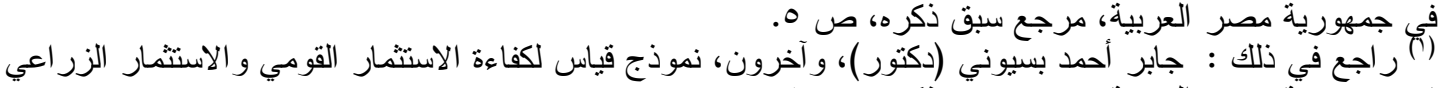

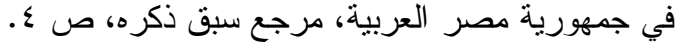


Assiut J. Agric. Sci., (45) No. (2) 2014 (197-216) (Special Issue)

(The $7^{\text {th }}$ Conference of Young Scientists) Fac. of Agric. Assiut University April, 28, 2014

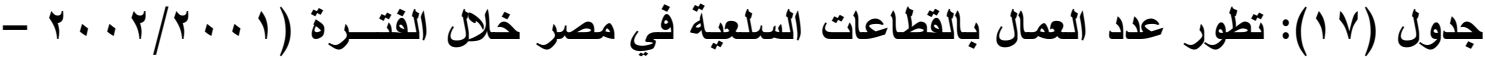

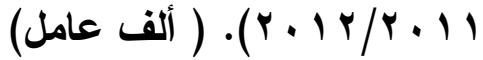

\begin{tabular}{|c|c|c|c|c|c|}
\hline التشييل والبناء & قطاع الكهرباء & قطاع البترول & قطاع الصناعة & قطاع الزراعة & السنو ات \\
\hline ITH & ITs,. & or, & $r \| r,$. & $\varepsilon q V Y$ & $r \ldots r / r \ldots l$ \\
\hline DTH TMO & ITI,. & 00,0 & YIV0,0 & $0.11,7$ & $r \ldots r / r \ldots r$ \\
\hline $1 \leq .0$ & $1 r 9,0$ & $71, r$ & YrOE, & $0.1 \varepsilon$ & $r \ldots \varepsilon / r \ldots r$ \\
\hline $1 \leqslant \mu V$ & $1 \leqslant \varepsilon, 1$ & $7 \wedge, 1$ & דוצדו & $010 \mathrm{~V}$ & $r \ldots 0 / r \ldots \varepsilon$ \\
\hline $1 \leqslant \vee 0$ & $1 \leq 7, \wedge$ & $V 7,0$ & $r \leqslant T o,$. & OYK & $r \ldots T / r \ldots 0$ \\
\hline 10Y0 & $1 \leq 9,1$ & $9 \cdot, 5$ & TOYV,O & & $r \ldots v / r \ldots r$ \\
\hline 101. & $104,$. & $1.0,0$ & YYOY,O & $0 \leqslant Y V$ & $r \ldots \Lambda / r \ldots V$ \\
\hline $17 \leq$. & $107, \pi$ & $|Y|, \cdot$ & 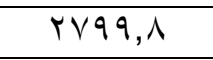 & $00 \leqslant 0$ & $r \ldots q / r \ldots 1$ \\
\hline IVAO & ** r Y O, & IYA, & $r q q, 1$ & 079. & $r \cdot 1 \cdot / r \cdot . q$ \\
\hline $1 \wedge V$. & "ঙ八,. & 1 To,. & ., & OVАร & $r .11 / r .1$. \\
\hline $190 \leqslant$ & MrT, & $|T|, 1$ & ". I, & $0 \wedge \leqslant 1$ & $* r .1 r / r .11$ \\
\hline $10 \vee 7, V$ & $190, r$ & $q \mu, Y Y$ & Trov, & orvi, s & المتوسط \\
\hline
\end{tabular}

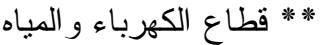

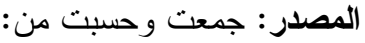

- - الجهاز المركزي للتعبئة العامة و الإحصاء، الكتاب الإحصائي السنوي، أعداد منقرقة.

- - البنك الأهلي المصري، النشرة الاقتصادية، أعداد مختلفة.

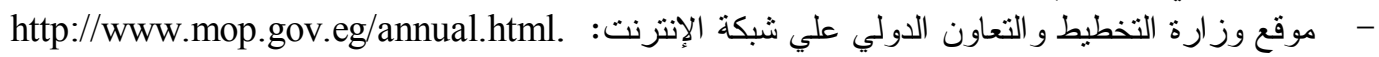

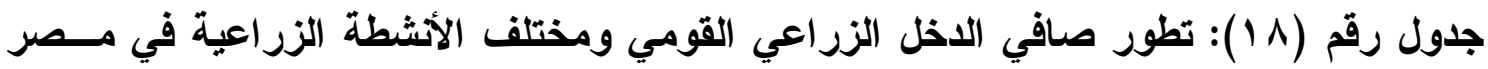

(بالأسعار الجارية وبالمليار جنيه)

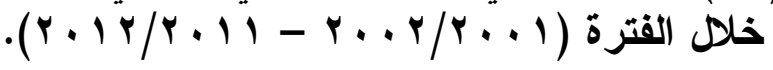

\begin{tabular}{|c|c|c|c|c|c|c|c|c|c|c|c|c|}
\hline \multicolumn{3}{|c|}{ الاخل السمكي } & \multicolumn{3}{|c|}{ الاخل الحيواني } & \multicolumn{3}{|c|}{ الاخل النباتي } & \multicolumn{3}{|c|}{ الاخل الزراعي القومي } & \multirow[b]{2}{*}{ السنوات } \\
\hline الأراضيدة & الأريمة & | الإجمالي | - ال & الألجيدة & |الأراضمي & | الإجمالي | - ال & الأرداضي & القدريمةّ & | الإجمالي & |الأراضيدة & الأراضمّي & | الإجمالي & \\
\hline$\cdot, 7$ & $\varepsilon, 9$ & 0,0 & $\cdot, r$ & 9,7 & $9, \wedge$ & $\vee, \varepsilon$ & T., & ऍ^, 乏 & $\wedge, 1$ & $\leqslant 0,0$ & T & $r \ldots r / r \ldots l$ \\
\hline$\cdot, 7$ & $0, \cdot$ & 0,7 & $\cdot, 1$ & $M$ & r & $\Lambda, r$ & rT, & $\leqslant 1,7$ & $9, \cdot$ & 01,0 & $7 ., 0$ & $r \ldots r / r \ldots r$ \\
\hline$\cdot, 7$ & 0,0 & 7,1 & $\cdot, 1$ & 10,0 & 10,7 & $9, \cdot$ & $r v, r$ & $\varepsilon\rceil, \wedge$ & $1 \cdot, \cdot$ & $0 \wedge, 7$ & $7 \wedge, 0$ & $r \ldots \varepsilon / r \ldots r$ \\
\hline $1, \cdot$ & $0, V$ & $7, V$ & $\cdot, V$ & 19,7 & $r \cdot r$ & $1 \cdot r$ & $\varepsilon 0, r$ & 00,0 & ir, & $v \cdot, 0$ & $\Lambda r, 0$ & $r \ldots o / r \ldots \varepsilon$ \\
\hline$\cdot, \varepsilon$ & $7, V$ & $v, 1$ & $\cdot, 1$ & $r_{0, \Lambda}$ & $r 0,9$ & $11, \wedge$ & $\varepsilon \wedge$, & $09, \wedge$ & $M, \varepsilon$ & $1 \cdot, 0$ & $q r, q$ & $r \ldots r / r \ldots o$ \\
\hline$\cdot, 0$ & $\wedge, \cdot$ & $\Lambda, 0$ & $\cdot, 0$ & $r V, r$ & $r V, V$ & IT,r & or, 9 & 77,1 & $1 \varepsilon, r$ & $\wedge \wedge, r$ & $1 \cdot r, \Sigma$ & $r \ldots v / r \ldots r$ \\
\hline$\cdot, 0$ & $9, \varepsilon$ & 9,9 & $\cdot, 7$ & $r q, 7$ & $r \cdot, r$ & 10,7 & $7 \cdot, 7$ & $V I, Y$ & 17,8 & 99,7 & $117, r$ & $r \ldots \lambda / r \ldots v$ \\
\hline$\cdot, \Sigma$ & $9, \varepsilon$ & $9, \wedge$ & $\cdot, 0$ & r,V & $r \varepsilon, r$ & 19,7 & $v r, l$ & $q r, v$ & $r \cdot, T$ & 117,1 & ITr,V & $r \ldots q / r \ldots 1$ \\
\hline$\cdot, r$ & $1 \cdot, \varepsilon$ & $1 \cdot, 7$ & $\cdot, 0$ & ro,. & ro,o & $r v, \varepsilon$ & $7 \varepsilon, 0$ & 91,9 & rA, l & $11 \cdot, \cdot$ & $\mid r \wedge, 1$ & $r \cdot 1 \cdot / r \cdot . q$ \\
\hline$\cdot, 0$ & $I T, V$ & IT, & $\cdot, 7$ & $r v, 7$ & $r \wedge, r$ & $r \wedge, T$ & $v \cdot, V$ & $99, r$ & $r q, \vee$ & $|Y|, \cdot$ & $10 \cdot, \mathrm{V}$ & $r .11 / r .1$. \\
\hline$\cdot, 7$ & $1 \leqslant, V$ & $10, r$ & $1, \cdot$ & $r \varepsilon, V$ & $r o, v$ & $r v, 0$ & $91, r$ & $\mid r \wedge, \vee$ & $r q, 1$ & $1 \leq \cdot, 7$ & $1 \vee 9, \vee$ & $r \cdot 1 r / r \cdot 11$ \\
\hline
\end{tabular}

المصدر: وزارة الزراعة واستصلاح الأراضي ، قطاع الثئون الاقتصادية ، الإدارة المركزية للتخطيط الزر اعي، الدخل

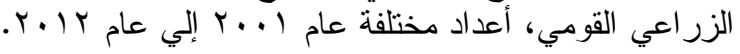




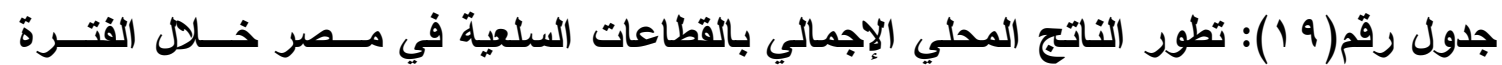

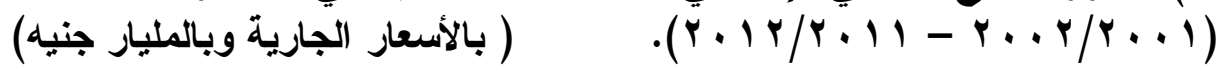

\begin{tabular}{|c|c|c|c|c|c|c|}
\hline قطاع التشييد & قالكهرباءع & قالبترول & قالصناعة & قالزراعة & الإاتج المحليلي & السنوات \\
\hline 10,1 & $0, r$ & $r q, r$ & $7 \pi, 0$ & 00,1 & TrT,O & $r \ldots r / r \ldots l$ \\
\hline 17,7 & 0,9 & ri, & $V Y, Y$ & $0 \wedge, \varepsilon$ & Tos, T & $r \ldots r / r \ldots r$ \\
\hline $17, V$ & $7, \Sigma$ & $\varepsilon r, r$ & $\wedge \mu, \tau$ & $\pi, \Lambda$ & $\leqslant 07, \Gamma$ & $r \ldots \varepsilon / r \ldots r$ \\
\hline 11,0 & 7,9 & $O V, \Sigma$ & $9 \cdot, \cdot$ & $79, r$ & $0.7,0$ & $r \ldots o / r \ldots \varepsilon$ \\
\hline$r \cdot, 1$ & $\vee, \Lambda$ & $7 \varepsilon$, & $q \wedge, \vee$ & $V 0, r$ & $0 \wedge 1,$. & $r \ldots T / r \ldots o$ \\
\hline rT, & 1,9 & $\wedge q, \wedge$ & $11 \varepsilon, 0$ & $\wedge 1, \wedge$ & $V) \cdot, \varepsilon$ & $r \ldots V / r \ldots r$ \\
\hline$r \cdot, r$ & 9,9 & ITH,V & $119,$. & $11 r$, & $100, \Gamma$ & $r \ldots \wedge / r \ldots V$ \\
\hline$\varepsilon \varepsilon$, & $\pi$, & $1 \leqslant \Lambda$, & $17 \leqslant, 0$ & $1 \Gamma 0,0$ & $99 \varepsilon$, & $r \ldots q / r \ldots 1$ \\
\hline or, 7 & $1 \leqslant, 9$ & $170, \mathrm{~V}$ & $19 \varepsilon$, & $171, \cdot$ & $110 \cdot, 7$ & $r \cdot 1 \cdot / r \cdot . q$ \\
\hline $7 \cdot, \cdot$ & 17,1 & 190, & YIT, & $19 \cdot, \cdot$ & $|r| \cdot, \cdot$ & $r .11 / r \cdot 1$. \\
\hline $7 V, \varepsilon$ & 19,1 & rYA, & YrA, & Y\, • & $1 \leq \vee 0$, & $* r \cdot 1 r / r \cdot 11$ \\
\hline
\end{tabular}

المصدر : جمعت وحسبت من : المنبت

- - البنك الأهلي المصري، النشرة الاقتصادية، أعداد مختلفة.

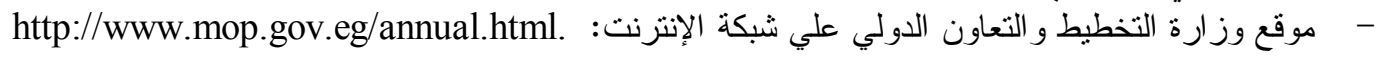

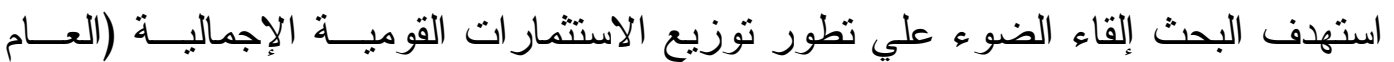

الملخص:

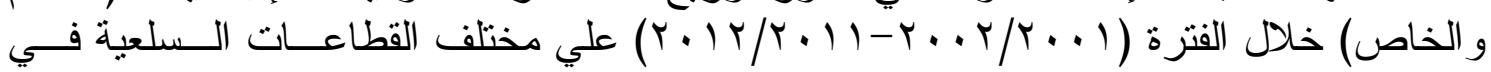

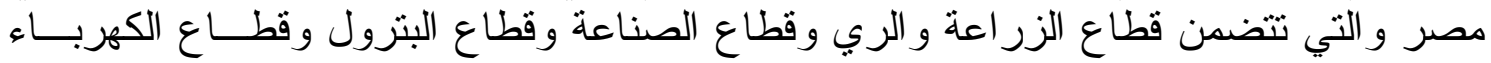

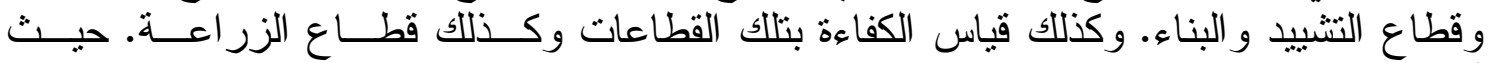

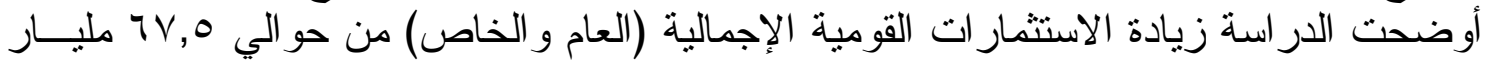

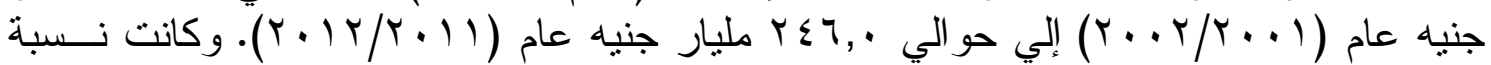

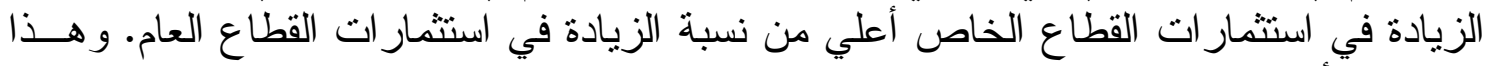

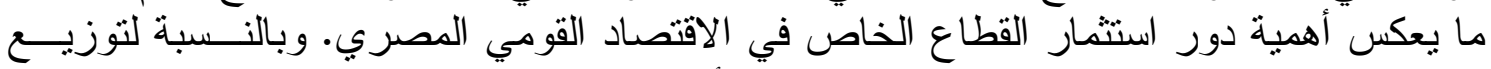

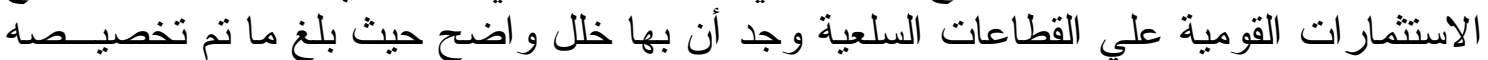

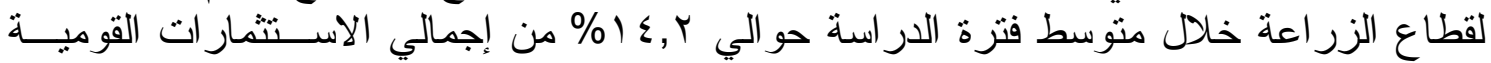

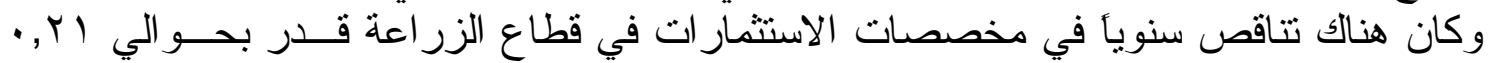

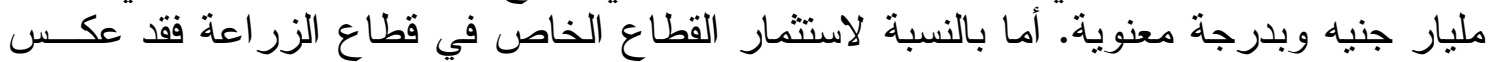

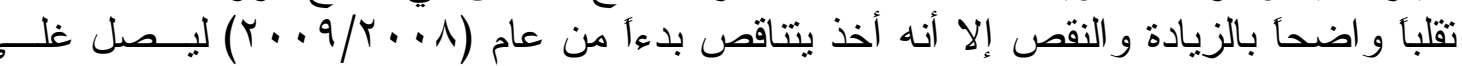

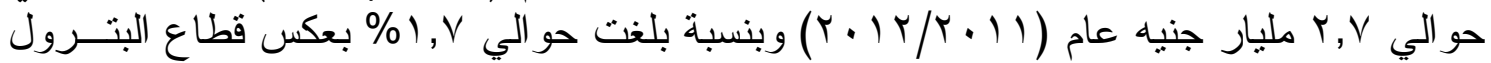

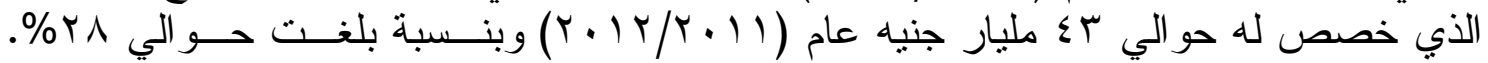

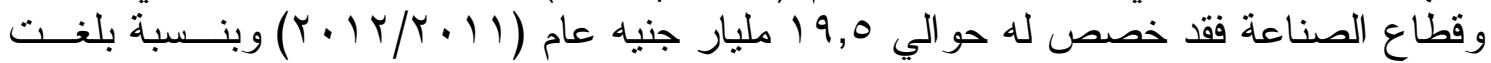

وقد عكست النتائج احتلال قطاع الزر اعة للمركز الأول بالنسبة لمعيــار متوســــ قيمـــة

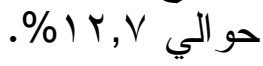
إنتاجية العامل. وكذللك بالنسبة لمعامل التوظف أو معامل رأس المال المستنثر بالنسبة للعامــلـ.

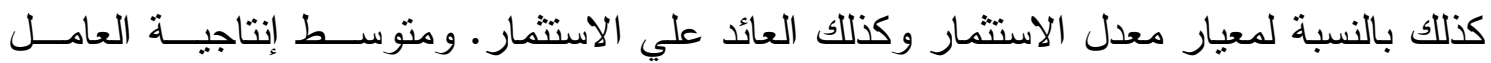

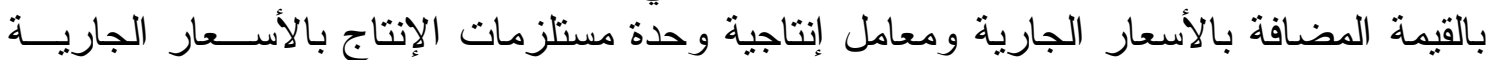

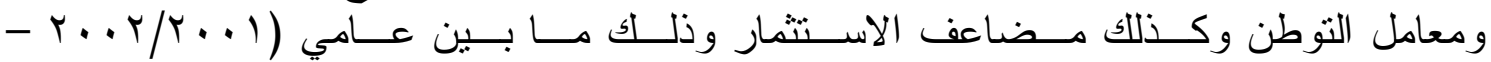

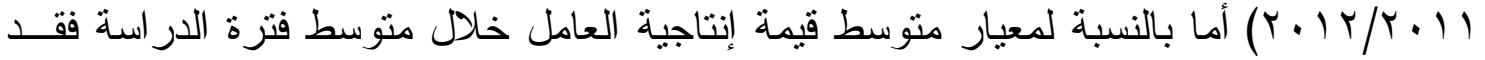


أحتل قطاع البترول المركز الأول ، تلاه قطاع الكهرباء ثم الصناعة، ثم الزر اعة و أخير ألتشييد و البناء.

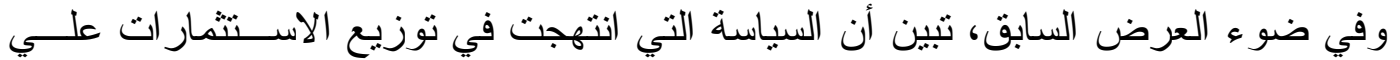

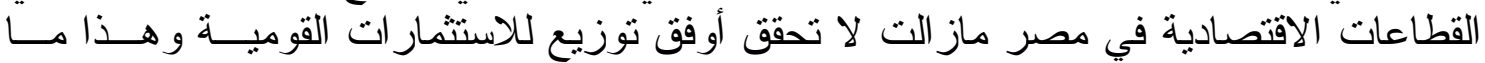

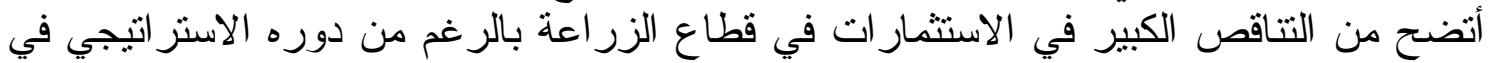

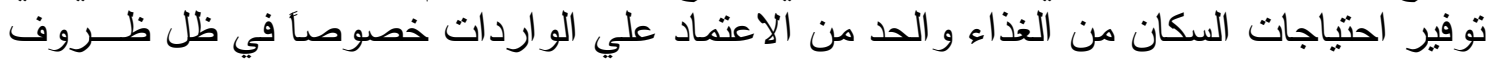

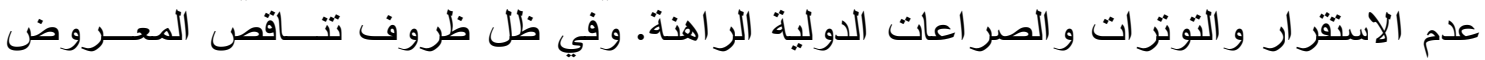

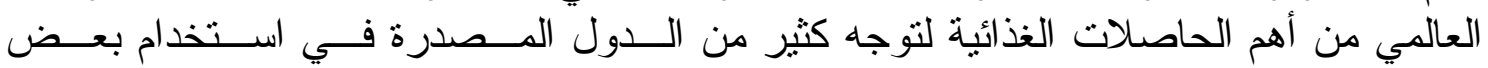
الحاصلات الغذائية في إنتاج الوقود الحيوي.

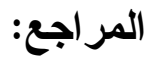

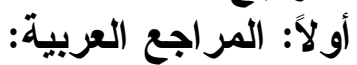

- أحمد محمود عبد العزيز، إنجازات ومعوقات الزعات التتمية الاقتصادية الزر اعية في مصر ، رسالة

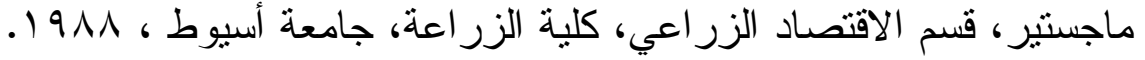

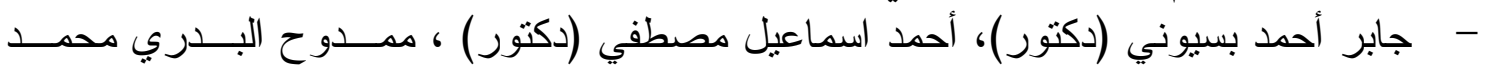

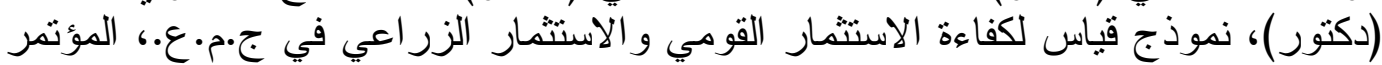

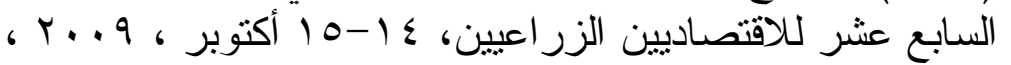

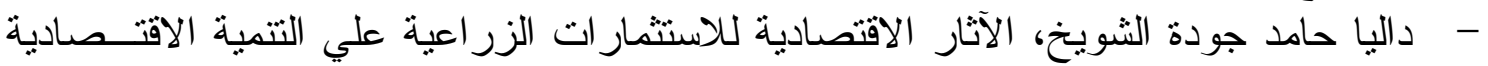

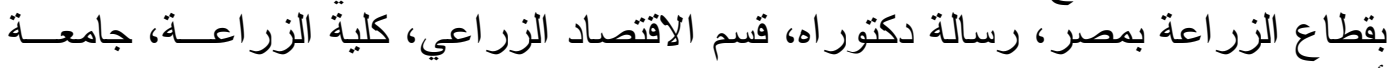

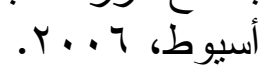

$$
\text { ثانياً: النشرات و الاوريات: }
$$

- - البنك الأهلي المصري، النشرة الاقتصادية، أعداد مختلفة.

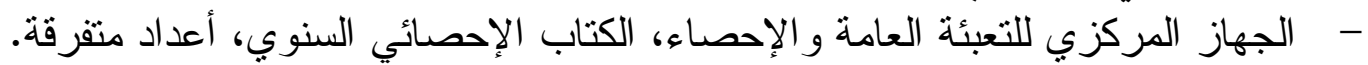

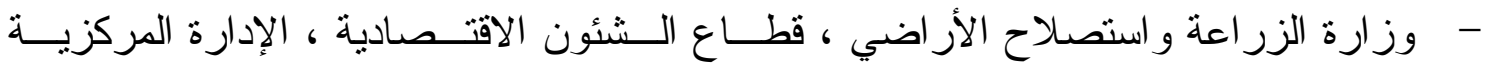

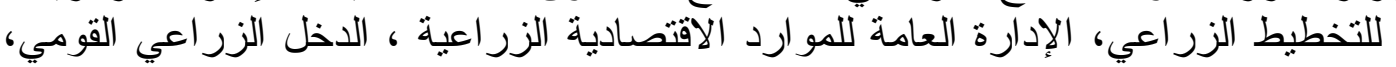

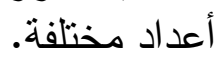

ثالثاً: مواقع عل شبكة الانترنت:

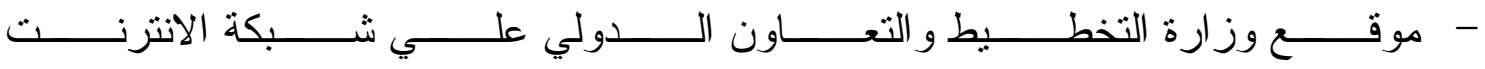
http://www.mop.gov.eg/annual.html 


\title{
Economic Study for Investment and Efficiency of Commodity Sectors
} in Egypt

Norhan Y. A. H. ; A. H. E. Sheemy ; T. H. Ismail and Y. A. Diab Agric. Econ. Dept., Fac. of Agric., Assiut Univ., Assiut.

\begin{abstract}
:
This research aims to set some lights on the development of the national investments (pubic and private) and its distribution between the commodity sectors in Egypt, during the period from 2001/2002 - 2011/2012.

The study found that there is a continuous decrease in the agricultural investments. Inspite of this the income from agricultural sector is more than that estimated from the other commodity sectors and realized high efficiency.

In this position the study recommends to redistribute the national investments between the economic sectors as well as increase the agricultural investments to realize real development in the Egyptian agriculture and at the national economy in general.
\end{abstract}

